\title{
Dissipative Numerical Method for a Flexible Euler-Bernoulli Beam with a Force Control in Rotation and Velocity Rotation
}

\author{
Bomisso G. Jean Marc ${ }^{1}$, Touré K. Augustin ${ }^{2}$ \& Yoro Gozo $^{1}$ \\ ${ }^{1}$ Université Nangui Abrogoua d'Abobo-Adjamé and UFR Sciences Fondamentales et Appliquées, Côte d'Ivoire \\ ${ }^{2}$ Institut National Polytechnique Houphouët-Boigny de Yamoussoukro, Côte d'Ivoire \\ Correspondence: Bomisso G. Jean Marc, Université Nangui Abrogoua d'Abobo-Adjamé and UFR Sciences Fondamen- \\ tales et Appliquées, BP 801 Abidjan 02, Côte d'Ivoire. E-mail: bogojm@yahoo.fr
}

Received: May 8, 2017 Accepted: June 5, 2017 Online Published: July 3, 2017

doi:10.5539/jmr.v9n4p30 URL: https://doi.org/10.5539/jmr.v9n4p30

\begin{abstract}
In this paper, we study a flexible Euler-Bernoulli beam clamped at one end and subjected to a force control in rotation and velocity rotation. We develop a finite element method, stable and convergent which preserves the property of time decay of energy in the continuous case. We prove firstly the existence and uniqueness of the weak solution. Then, we discretize the system in two steps: in the first step, a semi-discrete scheme is obtained for discretization in space and, in the second step, a fully-discrete scheme is obtained for discretization in time by the Crank-Nicolson scheme. At each step of the discretization, the a-priori error estimates are obtained.
\end{abstract}

Keywords: beam equation, Galerkin method, finite element methods, a priori estimates

\section{Introduction}

In this work, we study a dissipative numerical property by the finite element method for a flexible Euler-Bernoulli beams with a force control in rotation and velocity rotation. The dynamic system that models the mechanical phenomenon that changes over time is described as follows:

$$
\begin{array}{rlr}
w_{t t}(x, t)+w_{x x x x}(x, t)=0, & 0<x<1, t>0, \\
w(0, t)=w_{x}(0, t)=0, & t>0, \\
w_{x x x}(1, t)=0, & t>0, \\
w_{x x}(1, t)=-\alpha w_{x t}(1, t)-\beta w_{x}(1, t), & t>0 .
\end{array}
$$

$w(x, t)$ stands for the transverse displacement of the beam at the position $x$ and time $t$. The subscripts $t$ and $x$ denote derivatives with respect to time $t$ and position $x$ respectively. Moreover, $-w_{x x x x}(x, t) d x$ is the total lateral force acting on a slice of the beam of length $d x$, located at position $x$ and time $t$ and $w_{x x}(1, t)$ is the force in rotation acting on the rigid body from the beam at the time $t$. The nonnegative constants $\alpha$ and $\beta$ are the feedback gains that can be tuned in practice. For simplicity sake, the flexural rigidity function, the mass density function of the beam and the length of the beam are assumed to be unity. Moreover, the following notation $v=w_{t}$ (velocity of the beam) will be used in the sequel.

In (Touré, Koua \& Diop, 2016), it has been proved that the system (1)-(4) is well posed in the sense of $C_{0}$-semigroup of contractions. In order to perform the stability analysis of this system, the authors formulate the problem as an evolution problem first. Also, from Shkalikov's method (Shkalikov, 1986), a spectral analysis of the operator and the property of the Riesz basis were studied to derive the exponential stability of the system.

Furthermore, it should be noted that for all $t$, in our case, the total mechanical energy $\varepsilon: \mathbb{R}^{+} \rightarrow \mathbb{R}^{+}$of the system (1)-(4) is given by

$$
\varepsilon(t)=\frac{1}{2} \int_{0}^{1} w_{x x}^{2} d x+\frac{1}{2} \int_{0}^{1} v^{2} d x+\frac{\beta}{2}\left(w_{x}(1, t)\right)^{2}
$$

which decreases over time. Indeed, the time derivative of the energy functional $\varepsilon(t)$ along the classical solutions of (1)-(4) read as follows:

$$
\frac{d}{d t} \varepsilon(t)=-\alpha\left[v_{x}(1, t)\right]^{2} \leq 0
$$

because $\alpha \geq 0$. The right hand side of (6) serves as a motivation in the design of the control $\alpha w_{x t}(1, t)+\beta w_{x}(1, t)$, ensures the energy decay of the system in time. Moreover, according to Theorem 3.5 of (Touré, Koua \& Diop, 2016) whose proof is based on an idea of (Guo, 2002), the system (1)-(4) is exponentially stable for any $\beta>0$ and $\alpha \geq 0$. 


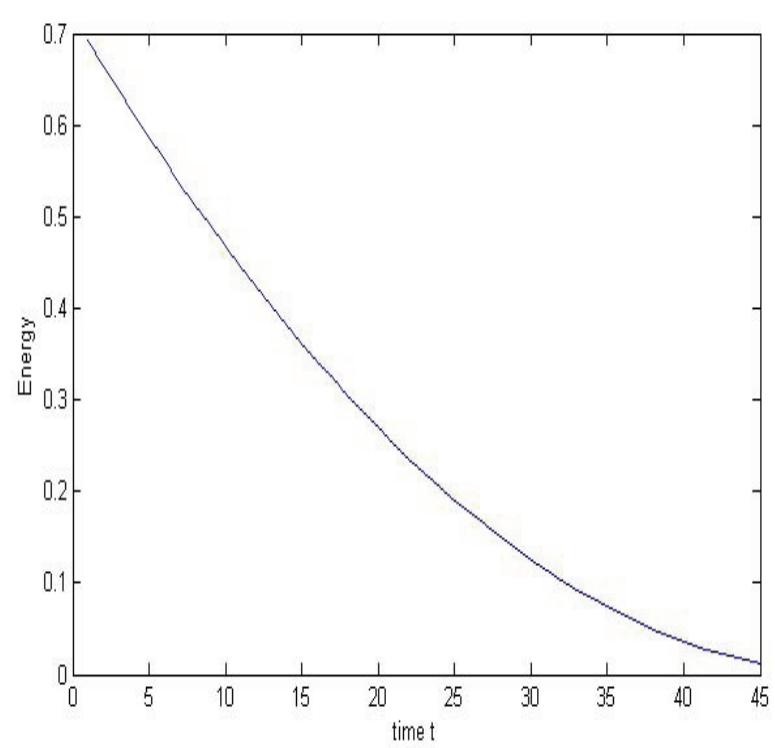

Figure 1. Energy decay

Our main contribution is to develop a convergent numerical method which faithfully reproduces some properties of this problem such as stability and energy decay.

The rest of the paper is organized as follows. In section 2, from the weak formulation, we show the existence, uniqueness and higher regularity of the weak solution. In section 3 and in section 4, we develop by finite element method, a numerical method for the system (1)-(4) which conserves the dissipativity property.

\section{Existence, Uniqueness and Higher Regularity of the Weak Solution}

\subsection{Formulation as a Dissipative Evolution Equation}

Let us introduce the following spaces:

$$
H^{m}(0,1)=\left\{w:[0,1] \rightarrow \mathbb{R} \mid w, w^{(1)}=\frac{\partial w}{\partial t}, \ldots, w^{(m)}=\frac{\partial^{m} w}{\partial t^{m}} \in L^{2}(0,1)\right\}
$$

where

$$
L^{2}(0,1)=\left\{w:\left.[0,1] \rightarrow \mathbb{R}\left|\int_{0}^{1}\right| w\right|^{2} d x<\infty\right\} .
$$

Then, we also introduce the following functional space:

$$
V=\left\{w \in H^{2}(0,1) \mid w(0)=w_{x}(0)=0\right\}
$$

and for energy space, the following hilbert space :

$$
\chi=\left\{y=(w, v)^{T}: w \in V, v \in L^{2}(0,1)\right\}=V \times L^{2}(0,1),
$$

where the superscript $T$ stands for the transpose. In the space $\chi$, we define the inner-product:

$$
<y, \widehat{y}>=\frac{1}{2} \int_{0}^{1}\left[w_{x x} \widehat{w}_{x x}+\widehat{v v}\right] d x+\frac{\beta}{2} w_{x}(1) \widehat{w}_{x}(1)
$$

where $y=(w, v)^{T} \in \chi$ and $\widehat{y}=(\widehat{w}, \widehat{v})^{T} \in \chi$. We denote by $\|.\|_{\chi}$ the associated norm. Next, we define an unbounded linear operator $A: D(A) \subset \chi \rightarrow \chi$ as follows:

$$
A(w, v)=\left(v,-w_{x x x x}\right)
$$

where $D(A)$, the domain of operator $A$ is as follows

$$
D(A)=\left\{(w, v) \in \chi: w \in\left(H^{4}(0,1) \cap V\right), v \in V, w_{x x x}(1)=0, w_{x x}(1)=-\alpha v_{x}(1)-\beta w_{x}(1)\right\} .
$$


Now we can write our problem as a first order evolution problem

$$
\left\{\begin{array}{l}
\frac{d}{d t} y(t)=A y(t) \\
y(0)=y_{0} \in \chi
\end{array}\right.
$$

where $y(t)=(w(., t), v(., t))^{T}, y(0)=\left(w_{0}, v_{0}\right)^{T}$ for all $t>0$.

We recall here the following fundamental well-posedness result obtained in Theorem 2.1 pp. 36 of (Touré, Koua \& Diop, 2016).

Theorem 1 The operator $A$, defined by (10) and (11), generates a $C_{0}$-semigroup of contractions on $\chi$ denoted by $\{S(t)\}_{t \geq 0}$.

Next results follow directly from Theorem 1:

Theorem 2 (12) has a unique mild solution $y(t)=S(t) y_{0} \in C([0, \infty) ; \chi)$ for all $y_{0} \in \chi$.

Notice that the contractivity of the semigroup also implies that $\|.\|_{\chi}$ is a good candidate for the Lyapunov functional for (12). Let the functional $\ell: \chi \rightarrow \mathbb{R}$ defined as follows

$$
\ell(y)=\|y\|_{\chi}^{2}=\frac{1}{2} \int_{0}^{1} w_{x x}^{2} d x+\frac{1}{2} \int_{0}^{1} v^{2} d x+\frac{\beta}{2}\left(w_{x}(1, t)\right)^{2} .
$$

Analogously as in (6), for all classical solutions $y$ it follows that:

$$
\frac{d}{d t} \ell(y)=\frac{d}{d t}\|y\|_{\chi}^{2}=-\alpha w_{t x}^{2}(1) \leq 0,
$$

hence time evolution of the Lyapunov functional along the classical solutions is non-increasing. Furthermore, from Theorem 1, the decay of energy along the classical solutions can be extended to mild solutions :

Theorem 3 Assume that $y(t)$ is the mild solution of (12) for all $y_{0} \in \chi$. Then $y(t) \rightarrow 0$ in $\chi$ when $t \rightarrow \infty$.

Remark 1 The right hand side of (14) is formed by a control variable. Thus, $\frac{d}{d t}\|y\|_{\chi}^{2}=0$ does not imply $y=0$ through (12).

Now write the system of equations (1)-(4) in the weak form.

\subsection{Weak Formulation}

Let $\phi \in V$. Multiplying (1) by $\phi$, integrating over [0,1] and taking into account the given boundary conditions (2)-(4), we have

$$
\int_{0}^{1} w_{t t} \phi d x+\int_{0}^{1} w_{x x} \phi_{x x} d x+\left[\beta w_{x}(1, t)+\alpha w_{t x}(1, t)\right] \phi_{x}(1)=0
$$

$\forall \phi \in V, t>0$.

It seeks to define a weak solution of (1)-(4). But first, make an appropriate choice of spaces. We follow an idea used in (Banks \& Rosen, 1987). Let the Hilbert space $Y=\mathbb{R}^{2} \times L^{2}(0,1)$ with the following inner product:

$$
<\eta, \xi>=<\eta_{(1)}, \xi_{(1)}>_{L^{2}}+\eta_{(2)} \xi_{(2)}+\eta_{(3)} \xi_{(3)},
$$

for all $\eta=\left(\eta_{(1)}, \eta_{(2)}, \eta_{(3)}\right), \xi=\left(\xi_{(1)}, \xi_{(2)}, \xi_{(3)}\right) \in Y$. We also define the following Hilbert space $X=\mathbb{R}^{2} \times V=\{y=$ $\left.\left(w(1), w_{x}(1), w\right) ; w \in V\right\}$ with the inner product

$$
<y_{1}, y_{2}>_{X}=<\left(w_{1}\right)_{x x},\left(w_{2}\right)_{x x}>_{L^{2}} .
$$

It can easily be shown that $X$ is densely embedded in $Y$ and suppose that the canonical injection of $X$ into $Y$ is continuous. Therefore, taking $Y$ as a pivot space we obtain a Gelfand triple :

$$
X \subset Y \subset X^{\prime}
$$

where $X^{\prime}$ is the dual of $X$. Consider the following bilinear forms:

$a_{1}: X \times X \rightarrow \mathbb{R} \quad\left(y_{1}, y_{2}\right) \mapsto a_{1}\left(y_{1}, y_{2}\right)=<y_{1}, y_{2}>_{X}+\beta\left(w_{1}\right)_{x}(1)\left(w_{2}\right)_{x}(1)$

$a_{2}: Y \times Y \rightarrow \mathbb{R} \quad(\eta, \xi) \mapsto \alpha \eta_{(2)} \xi_{(2)}$.

In the following definition, the bilinear form $<\ldots,>_{X, X^{\prime}}$ is the duality pairing between $X$ and $X^{\prime}$, which is a natural extension of the inner product in $Y$. 
Definition 4 Let $T>0$ be fixed. We say that $\widehat{w}=\left(w(1), w_{x}(1), w\right)$ is a weak solution of problem (1)-(4) on [0,1] if $\widehat{w} \in L^{2}(0, T ; X) \cap H^{1}(0, T ; Y) \cap H^{2}\left(0, T ; X^{\prime}\right)$ and satisfies

$$
<\widehat{w}_{t t}, \widehat{\phi}>_{X, X^{\prime}}+a_{1}(\widehat{w}, \widehat{\phi})+a_{2}\left(\widehat{w}_{t}, \widehat{\phi}\right)=0
$$

for almost everywhere on $t \in(0, T)$ and for all $\widehat{\phi} \in X$, with the following initial conditions

$$
\begin{gathered}
\widehat{w}(0)=\widehat{w}_{0}=\left(w_{0}(1),\left(w_{0}\right)_{x}(1), w_{0}\right) \in X \\
\widehat{w}_{t}(0)=\widehat{v}_{0}=\left(v_{0}(1),\left(v_{0}\right)_{x}(1), v_{0}\right) \in Y .
\end{gathered}
$$

Remark 2 The formulation (18) is equivalent to equality (15) if $w \in H^{2}(0, T, X)$. Furthermore, in the expression (19), the first two components of the right hand side are the boundary traces of $w_{0} \in V$. But for $\left(v_{0}\right)(1)$ and $\left(v_{0}\right)_{x}(1)$ in $(20)$, they are given in addition to the function $v_{0}$ and not as its trace. Here, the term $u_{t x}(1)$ also need to be considered. Then, the bilinear form $a_{2}(.,$.$) with the first order boundary term in t$ requires a slight generalization of the standard theory (as presented for example in chapter 3 section 8 of (Lions \& Magenes, 1968) or again in section 7.2 of (Evans, 1998)).

Recall the following Lemmas provided in Theorems 3.1 pp. 23 and 6.2 pp. 34 of (Lions \& Magenes, 1968) where the definition of the intermediate spaces is given and which will be useful in proving theorem of existence of weak solution.

Notice that, in the following, $[X, Y]_{\theta}$, with $0 \leq \theta \leq 1$ is the intermediate space defined as in chapter 1 pp. $11-13$ of (Lions \& Magenes, 1968), for $X$ and $Y$ Hilbert spaces, $X \subset Y, X$ dense in $Y$ with continuous injection by means of domains of positive self-adjoint operators. Remark also that for $\theta=0,[X, Y]_{0}=X$ and for $\theta=1,[X, Y]_{1}=Y$.

Lemma 5 Let $X$ and $Y$ be two Hilbert spaces, such that $X$ is dense and continuously embedded in $Y$. Assume that $w \in L^{2}(0, T ; X)$ and $v \in L^{2}(0, T ; Y)$. Then $w \in C\left([0, T] ;[X, Y]_{\frac{1}{2}}\right)$, after, possibly, a modification on a set of measure zero.

Since $X \subset[X, Y]_{\theta} \subset Y$, each space being dense in the following, we have by duality (without any identification between space and its dual) for $\theta \in] 0,1[$ :

$$
Y^{\prime} \subset[X, Y]_{\theta}^{\prime} \subset X^{\prime},
$$

each space being dense in the following. We have the following duality theorem:

Lemma 6 Let $X$ and $Y$ be two Hilbert spaces, such that $X$ is dense and continuously embedded in $Y$. For all $\theta \in] 0,1[$, $[X, Y]_{\theta}^{\prime}=\left[Y^{\prime}, X^{\prime}\right]_{1-\theta}$ holds.

We will also use the following result:

Theorem 7 Let $V$ be a subspace of $H^{2}(0,1)$. Then there exists a infinite sequence of functions $\left\{\phi_{i}\right\}_{i=1}^{\infty}$ such that

$$
\left\{\phi_{i}\right\}_{i=1}^{\infty} \text { is an orthogonal basis of } V
$$

and

$$
\left\{\phi_{i}\right\}_{i=1}^{\infty} \text { is an orthonormal basis of } L^{2}(0,1) .
$$

Proof. Let the operator $B: V \rightarrow L^{2}(0,1)$ defined as

$$
\forall w \in V, \quad B w=w_{x x x x} .
$$

Consider the following boundary value problem :

$$
\left\{\begin{array}{l}
B w(x)=f(x), \quad x \in(0,1) \\
w(0)=w_{x}(0)=w_{x x}(1)=w_{x x x}(1)=0 .
\end{array}\right.
$$

Assume that $f \in L^{2}(0,1)$. Let $\phi \in V$. Multiplying by $\phi$, integrating twice by parts and taking into account the given boundary conditions yields:

$$
\int_{0}^{1} w_{x x} \phi_{x x} d x=\int_{0}^{1} f \phi d x, \quad \forall \phi \in V .
$$

The fact that $f \in L^{2}(0,1)$ ensures the continuity of the linear form. Set

$$
a_{3}(w, \phi)=\int_{0}^{1} w_{x x} \phi_{x x} d x .
$$


Then $a_{3}$ is symmetric bilinear form, bounded and coercive on $V$. The Lax-Milgram theorem allows us to conclude the existence and uniqueness of the solution $w$ of (21). Then, there exists a unique weak solution $w$ in $V$ such that $w=B^{-1}(f)$ with $B^{-1}: L^{2}(0,1) \rightarrow L^{2}(0,1)$. We remark that $B^{-1}$ is obviously linear and bounded. Furthermore, there exists a constant $C>0$ such that

$$
\|w\|_{H^{2}(0,1)} \leq C\|f\|_{L^{2}(0,1)}
$$

and since $V$ is compactly embedded in $L^{2}(0,1)$ then $B^{-1}$ is compact. It remains to show that it's symmetric. Let $f, g \in$ $L^{2}(0,1)$ and denote $w=B^{-1}(f)$ and $v=B^{-1}(g)$. By straightforward calculation and integration by parts, we finally obtain

$$
<B^{-1} f, g>_{L^{2}(0,1)}=\int_{0}^{1} v_{x x} w_{x x} d x=a_{3}(v, w) .
$$

Analogously, we get

$$
<f, B^{-1} g>_{L^{2}(0,1)}=\int_{0}^{1} w_{x x} v_{x x} d x=a_{3}(w, v) .
$$

Thus, $a_{3}$ is symmetric and we have

$$
<B^{-1} f, g>_{L^{2}(0,1)}=<f, B^{-1} g>_{L^{2}(0,1)} .
$$

Hence $B^{-1}$ is symmetric. In view of compactness and symmetry properties of the bounded linear operator $B^{-1}$, there exists a countable orthonormal basis $\left\{\phi_{i}\right\}_{i=1}^{\infty}$ of $L^{2}(0,1)$ constitued of eigenvectors $B^{-1}$. Furthermore, these eigenvectors are functions of $V$ according to definition of $B^{-1}$. Moreover, from the weak formulation, one can see that the basis $\left\{\phi_{i}\right\}_{i=1}^{\infty}$ is an orthogonal basis of $V$ with respect to the inner product $a_{3}(.$, .).

\subsection{Existence of the Weak Solution}

Theorem 8 There exists a weak solution $\widehat{w}$ of the equivalent weak formulation (18) such that :

$$
\begin{gathered}
\widehat{w} \in L^{\infty}(0, T ; X), \widehat{w}_{t} \in L^{\infty}(0, T ; Y), \\
\widehat{w} \in C\left([0, T] ;[X, Y]_{\frac{1}{2}}\right), \\
\widehat{w}_{t} \in C\left([0, T] ;[X, Y]_{\frac{1}{2}}^{\prime}\right) .
\end{gathered}
$$

Proof. This proof is based on the Faedo-Galerkin's method and is an adaption of the proof of Theorem 8.1 pp. 287-290 in (Lions \& Magenes, 1968). According to Theorem 7, there exists by extension an infinite sequence of functions $\left\{\widehat{\phi}_{i}\right\}_{i=1}^{\infty}$ that is an orthogonal basis for $X$ and an orthonormal basis for $Y$. Consider such a sequence. Introduce the following finite dimensional spaces spanned by $\left\{\widehat{\phi}_{i}\right\}_{i=1}^{m}$ defined as:

$$
\forall m \in \mathbb{N}, \quad \widehat{V}_{m}:=\operatorname{span}\left\{\widehat{\phi}_{1}, \ldots, \widehat{\phi}_{m}\right\}=\left\{\sum_{j=1}^{m} \alpha_{j} \widehat{\phi}_{j}, \alpha_{1}, \alpha_{2}, \ldots, \alpha_{m} \in \mathbb{R}\right\} .
$$

Step 1 (Construction of approximate solutions): We seek $\widehat{w}=\widehat{w}_{m}(t) \in \widehat{V}_{m}$ the approximate solution of the problem. Then $\widehat{w}$ is in the form:

$$
\widehat{w}_{m}=\sum_{i=1}^{m} g_{i m}(t) \widehat{\phi}_{i},
$$

where $g_{i m}(t) \in \mathbb{R}(0 \leq t \leq T, i=1, \ldots, m)$ are solutions of the formulation (18) on $\widehat{V}_{m}$. For a fixed $m \in \mathbb{N}$, we have:

$$
<\left(\widehat{w}_{m}\right)_{t t}, \widehat{\phi}>_{Y}+a_{1}\left(\widehat{w}_{m}, \widehat{\phi}\right)+a_{2}\left(\left(\widehat{w}_{m}\right)_{t}, \widehat{\phi}\right)=0 \quad \forall \widehat{\phi} \in \widehat{V}_{m} .
$$

The approximate differentials equations system (28) is completed with the initial conditions:

$$
\begin{aligned}
& \widehat{w}_{m}(0)=\widehat{w}_{m 0}, \widehat{w}_{m 0}=\sum_{i=1}^{m} \alpha_{i m} \widehat{\phi}_{i} \rightarrow \widehat{w}_{0} \text { in } X \text { when } m \rightarrow \infty \\
& \widehat{v}_{m}(0)=\widehat{v}_{m 0}, \widehat{v}_{m 0}=\sum_{i=1}^{m} \beta_{i m} \widehat{\phi}_{i} \rightarrow \widehat{v}_{0} \text { in } Y \text { when } m \rightarrow \infty,
\end{aligned}
$$

with $\alpha_{i m}=g_{i m}(0)$ and $\beta_{i m}=\left(g_{i m}\right)_{t}(0)$. According to standard existence theory for ordinary differential equations, we are insured of the existence of solution $\widehat{w}_{m} \in C^{2}([0, T] ; X)$ of (28)-(30) for $0 \leq t \leq T$. 
Step 2 (A-priori estimates on approximate solutions): Let $E: \mathbb{R} \times X \rightarrow \mathbb{R}$ be the analogue of the Lyapunov functional as defined by (13):

$$
\begin{gathered}
E(t, \widehat{w})=\frac{1}{2}\left[\int_{0}^{1} \widehat{w}_{t}^{2} d x+\int_{0}^{1} \widehat{w}_{x x}^{2} d x+\beta \widehat{w}_{(2)}^{2}(t)\right] \\
E(t, \widehat{w})=\frac{1}{2}\left\|\widehat{w}_{t}(t)\right\|_{Y}^{2}+\frac{1}{2}\|\widehat{w}(t)\|_{X}^{2}+\frac{\beta}{2}\left(\widehat{w}_{(2)}(t)\right)^{2} . \\
E(t, \widehat{w})=\|(w, v)\|_{X} .
\end{gathered}
$$

Assuming that there exists a solution $\widehat{w}_{m} \in C^{2}\left([0, \tau] ; \widehat{V}_{m}\right)$ to (28) on some interval $[0, \tau]$ and taking $\widehat{\phi}=\left(\widehat{w}_{m}\right)_{t}$ in (28), a straightforward calculation yields

$$
\frac{d}{d t} E\left(t, \widehat{w}_{m}\right)=-\alpha\left[\left\{\left(\widehat{w}_{m}\right)_{(2)}\right\}_{t}(t)\right]^{2} \leq 0,
$$

for all $t \in[0, \tau]$. Dissipation of the functional $E$ corresponds to the decay in (14) for the classical solution. This implies uniform boundedness of the solution on $[0, \tau]$ :

$$
E\left(t, \widehat{w}_{m}\right) \leq E\left(0, \widehat{w}_{m 0}\right), t \geq 0
$$

which implies that

$$
\begin{gathered}
\left\{\widehat{w}_{m}\right\}_{m \in \mathbb{N}} \text { is bounded in } C([0, T] ; X), \\
\left\{\left(\widehat{w}_{m}\right)_{t}\right\}_{m \in \mathbb{N}} \text { is bounded in } C([0, T] ; Y) .
\end{gathered}
$$

It remains to find set in which $\left(\widehat{w}_{m}\right)_{t t}$ is bounded. Considering the results (34)-(35), it is shown that for all $\widehat{\phi} \in X$ :

$$
\left|a_{1}\left(\widehat{w}_{m}(t), \widehat{\phi}\right)+a_{2}\left(\left(\widehat{w}_{m}\right)_{t}(t), \widehat{\phi}\right)\right| \leq M\|\widehat{\phi}\|_{X}, \forall t \in[0, T]
$$

where $M$ is a positive constant which does not depend on $m$. Now, let $m \in \mathbb{N}$ be fixed. Furthermore, let $\widehat{\phi} \in X$ and $\widehat{\phi}=\widehat{\varphi}_{1}+\widehat{\varphi}_{2}$ such that $\widehat{\varphi}_{1} \in \widehat{V}_{m}$ and $\widehat{\varphi}_{2}$ orthogonal to $\widehat{V}_{m}$ in $Y$. Then, we get $<\left(\widehat{w}_{m}\right)_{t t}, \widehat{\phi}>_{Y}=<\left(\widehat{w}_{m}\right)_{t t}, \widehat{\varphi}_{1}>_{Y}$. From (28) and (36), we have:

$$
<\left(\widehat{w}_{m}\right)_{t t}, \widehat{\phi}>_{Y}=-a_{1}\left(\widehat{w}_{m}(t), \widehat{\varphi}_{1}\right)-a_{2}\left(\left(\widehat{w}_{m}\right)_{t}(t), \widehat{\varphi}_{1}\right) \leq M\left\|\widehat{\varphi}_{1}\right\|_{X} \leq M\|\widehat{\phi}\|_{X}
$$

This implies that

$$
\left(\widehat{w}_{m}\right)_{t t} \text { is bounded in } C\left([0, T] ; X^{\prime}\right) \text {. }
$$

Step 3 (Passage to the limit): According to the Eberlein-Šmulian Theorem (see Brezis, 2011, e.g.), we can extract weakly convergent subsequences $\left\{\widehat{w}_{m_{l}}\right\}_{l \in \mathbb{N}},\left\{\left(\widehat{w}_{m_{l}}\right)_{t}\right\}_{l \in \mathbb{N}}$ and $\left\{\left(\widehat{w}_{m_{l}}\right)_{t t}\right\}_{l \in \mathbb{N}}$ with $\widehat{w} \in L^{2}(0, T ; X), \widehat{w}_{t} \in L^{2}(0, T ; Y)$ and $\widehat{w}_{t t} \in L^{2}\left(0, T ; X^{\prime}\right)$ such that:

$$
\begin{gathered}
\left\{\widehat{w}_{m_{l}}\right\} \rightarrow \widehat{w} \text { in } L^{2}(0, T ; X), \\
\left\{\left(\widehat{w}_{m_{l}}\right)_{t}\right\} \rightarrow \widehat{w}_{t} \text { in } L^{2}(0, T ; Y), \\
\left\{\left(\widehat{w}_{m_{l}}\right)_{t t}\right\} \rightarrow \widehat{w}_{t t} \text { in } L^{2}\left(0, T ; X^{\prime}\right) .
\end{gathered}
$$

Furthermore, (40) yields

$$
\left\{\left(\widehat{w}_{(2) m_{l}}\right)_{t}\right\} \rightarrow \widehat{w}_{(2) t} \text { in } L^{2}(0, T ; \mathbb{R}) .
$$

Let $m_{0} \in \mathbb{N}$. For all functions $\widehat{\varphi} \in L^{2}\left(0, T ; \widehat{V}_{m_{0}}\right)$ of the form

$$
\widehat{\varphi}(t, x)=\sum_{j=1}^{m_{0}} \mu_{j}(t) \phi_{j}(x)
$$

where $\mu_{j} \in L^{2}(0, T ; \mathbb{R})$ and for all $m_{l} \geq m_{0}$, the formulation (28) becomes:

$$
\int_{0}^{T}<\left(\widehat{w}_{m_{l}}\right)_{t t}, \widehat{\varphi}>_{Y}+a_{1}\left(\widehat{w}_{m l}, \widehat{\varphi}\right)+a_{2}\left(\left(\widehat{w}_{m_{l}}\right)_{t}, \widehat{\varphi}\right) \mathrm{d} t=0 .
$$

Therefore, passing on to the limit in (44) for $m=m_{l}$, when $l \rightarrow \infty$ and using the convergence results (39)-(41), one obtains:

$$
\int_{0}^{T}<\widehat{w}_{t t}, \widehat{\varphi}>_{X, X^{\prime}}+a_{1}(\widehat{w}, \widehat{\varphi})+a_{2}\left(\widehat{w}_{t}, \widehat{\varphi}\right) d t=0 .
$$


Then, one obtains $<\widehat{w}_{t t}, \widehat{\varphi}>_{X, X^{\prime}}+a_{1}(\widehat{w}, \widehat{\varphi})+a_{2}\left(\widehat{w}_{t}, \widehat{\varphi}\right)=0$ a.e on $[0, T]$ for all $\widehat{\varphi} \in L^{2}(0, T ; X)$. Since the functions $\widehat{\varphi}$ of the form (43) are dense in $L^{2}(0, T ; X), \widehat{w}$ is therefore the solution of the weak formulation. For the additional regularity, from the construction of the weak solution and due to (34)-(35), $\widehat{w}$ satisfies (24). Furthermore $\widehat{w}$ satisfies (25) using the Lemma 5 , after, possibly a modification on a set of measure zero, and the regularity (26) follows from Lemma 5 and Lemma 6.

\subsection{Uniqueness of the Weak Solution}

Theorem 9 The solution $\widehat{w}$ of the weak formulation (18) with the initial conditions (19)-(20) is unique.

Proof. This proof of uniqueness is an adaption of the proof of Theorem 8.1 pp. 290-291 in (Lions \& Magenes, 1968). Before showing uniqueness, we prove that the solution $\widehat{w}$ satisfies the initial conditions (19)-(20). Let $\widehat{\phi} \in C^{2}([0, T] ; X)$ such that $\widehat{\phi}(T)=0$ and $\widehat{\phi}_{t}(T)=0$. Integrating the equation (18) over $[0, T]$, we get:

$$
\int_{0}^{T}\left[<\widehat{w}_{t t}, \widehat{\phi}>_{X, X^{\prime}}+a_{1}(\widehat{w}, \widehat{\phi})+a_{2}\left(\widehat{w}_{t}, \widehat{\phi}\right)\right] d \tau=0 .
$$

By integrating twice by parts over $[0, T]$ under the duality pairing, we have:

$$
\int_{0}^{T}\left[<\widehat{w}, \widehat{\phi}_{t t}>_{Y}+a_{1}(\widehat{w}, \widehat{\phi})+a_{2}\left(\widehat{w}_{t}, \widehat{\phi}\right)\right] d \tau=<\widehat{w}_{t}(0), \widehat{\phi}(0)>_{X, X^{\prime}}-<\widehat{w}(0), \widehat{\phi}_{t}(0)>_{Y} .
$$

Let $m$ be fixed. We obtain analogously by integrating twice by parts expression(28) :

$$
\int_{0}^{T}\left[<\widehat{w}_{m}, \widehat{\phi}_{t t}>_{Y}+a_{1}\left(\widehat{w}_{m}, \widehat{\phi}\right)+a_{2}\left(\left(\widehat{w}_{m}\right)_{t}, \widehat{\phi}\right)\right] d \tau=<\widehat{v}_{m 0}, \widehat{\phi}(0)>_{Y}-<\widehat{w}_{m 0}, \widehat{\phi}(0)>_{Y}
$$

Passing to the limit in (48) along the convergent subsequence, using (29)-(30) and (39)-(41), we obtain:

$$
\int_{0}^{T}\left[<\widehat{w}, \widehat{\phi}_{t t}>_{Y}+a_{1}(\widehat{w}, \widehat{\phi})+a_{2}\left((\widehat{w})_{t}, \widehat{\phi}\right)\right] d \tau=<\widehat{v}_{0}, \widehat{\phi}(0)>_{Y}-<\widehat{w}_{0}, \widehat{\phi}_{t}(0)>_{Y}
$$

Comparing expressions (47) with (49), we can deduce that $\widehat{w}_{0}=\widehat{w}(0)$ and $\widehat{w}_{t}(0)=\widehat{v}(0)$. Thus the initial conditions (19)(20) are satisfied.

Now it suffices to show that the only weak solution of (18) is $\widehat{w} \equiv 0$. To verify this, fix $0 \leq s \leq T$ and introduce an auxiliary function: $\widehat{\psi}:] 0, T[\rightarrow \mathbb{R}$,

$$
\widehat{\psi}(t)=\left\{\begin{array}{cl}
\int_{t}^{s} \widehat{w}(\tau) d \tau & 0<t<s \\
0 & t \geq s .
\end{array}\right.
$$

Integrating (18) over $[0, T$ [ and then using one integration by parts with $\widehat{\psi}(t)=\widehat{\phi}(t)$ in (18), we obtain:

$$
\begin{gathered}
\int_{0}^{s}\left[<\widehat{w}_{t}(\tau), \widehat{w}(\tau)>_{Y}-a_{1}\left(\widehat{\psi}_{t}(\tau), \widehat{\psi}(\tau)\right)+a_{2}(\widehat{w}(\tau), \widehat{w}(\tau))\right] d \tau=0 . \\
\int_{0}^{s} \frac{d}{d t}\left[\frac{1}{2}\|\widehat{w}(\tau)\|_{Y}^{2}-\frac{1}{2} a_{1}(\widehat{\psi}(\tau), \widehat{\psi}(\tau))\right] \mathrm{d} \tau=-\int_{0}^{s} a_{2}(\widehat{w}(\tau), \widehat{w}(\tau)) d \tau .
\end{gathered}
$$

This is equivalent to $\left[\frac{1}{2}\|\widehat{w}(\tau)\|_{X}^{2}-\frac{1}{2} a_{1}(\widehat{\psi}(\tau), \widehat{\psi}(\tau))\right]_{0}^{s}=-\int_{0}^{s} a_{2}(\widehat{w}(\tau), \widehat{w}(\tau)) \mathrm{d} \tau$. Thus we get $\frac{1}{2}\|\widehat{w}(s)\|_{X}^{2}+\frac{1}{2} a_{1}(\widehat{\psi}(0), \widehat{\psi}(0)) \leq 0$. Since the bilinear form $a_{1}(.,$.$) is coercive, \widehat{w}(s) \equiv 0$ and $\widehat{\psi}(0)=0$. Also, since $\left.s \in\right] 0 ; T[$ was arbitrary then $\widehat{w} \equiv$ 0 .

\subsection{Higher Regularity Results}

Before stating the theorem of the stronger continuity of the weak solution, recall the lemma 8.1 of chapter 3 pp. 297 of (Lions \& Magenes, 1968) which will be used in the proof of this theorem.

Lemma 10 Let $X$ and $Y$ two Banach spaces, $X \subset Y$ with continuous injection, the space $X$ being reflexive. We set:

$$
C_{w}([0, T] ; Y)=\left\{w \in L^{\infty}(0, T ; Y): t \mapsto<f, w(t)>\text { is continuous on }[0, T], \forall f \in Y^{\prime}\right\}
$$

which denotes the space of weakly continuous functions with values in $Y$. Thus we get

$$
L^{\infty}(0, T ; X) \cap C_{w}([0, T] ; Y)=C_{w}([0, T] ; X) .
$$


Proof. For the proof, the reader is referred to chapter 3 pp. 297-298 in (Lions \& Magenes, 1968).

Theorem 11 The weak solution $\widehat{w}$ of (18)-(20) satisfies

$$
\begin{aligned}
& \widehat{w} \in C([0, T] ; X), \\
& \widehat{w}_{t} \in C([0, T] ; Y),
\end{aligned}
$$

after possibly a modification on a set of measure zero.

Proof. This proof is an adaption of standard strategies in section 8.4 of (Lions \& Magenes, 1968) pp. 297-301 and in section 2.4 of (Temam, 1988). Using Lemma 10, it results from (26)-(27) that $\widehat{w} \in C_{w}([0, T] ; X)$. Furthermore, (24) and (26) imply $\widehat{w}_{t} \in C_{w}([0, T] ; Y)$.

Now, we use a common technique in functional analysis, specifically in distribution theory, to move from a problem of generalized functions to a restriction of regular functions easier to handle. Let a scalar cutoff function $\xi \in C^{\infty}$ ( $\mathbb{R}$ ) be fixed such that $\xi(x)=1$ if $x \in J \subset \subset[0, T]$ and $\xi(x)=0$ else. The function $\xi \widehat{w}$ is then compactly supported. Let $\eta^{\varepsilon}$ be a standard mollifier in time. For example, the function $\eta^{\varepsilon}$ may be given by $\eta^{\varepsilon}(t)=\varepsilon^{-1} \eta\left(\frac{t}{\varepsilon}\right)$ where

$$
\eta(t)= \begin{cases}C \exp \left[-1 /\left(1-|t|^{2}\right)\right], & |t|<1 \\ 0, & |t| \geq 1\end{cases}
$$

belongs to $C_{c}^{\infty}(\mathbb{R})$ for any $C$. We choose $C$ such that $\int_{\mathbb{R}} \eta d x=1$. Introduce the notation $\widehat{w}^{\varepsilon}=\eta^{\varepsilon} * \xi \widehat{w} \in C_{c}^{\infty}(\mathbb{R}, X)$. In addition, $\widehat{w}^{\varepsilon}$ converges to $\widehat{w}$ in $X$ and $\left(\widehat{w}^{\varepsilon}\right)_{t}$ converges to $\widehat{w}_{t}$ a.e in $H$ for all element on $J$. Hence, $\widehat{E}\left(t, \widehat{w}^{\varepsilon}\right)$ converges to $\widehat{E}(t, \widehat{u})$ a.e on $J$. Since $\widehat{w}^{\varepsilon}$ is smooth, a straightforward calculation on $J$ gives:

$$
\frac{d}{d t} E\left(t, \widehat{w}^{\varepsilon}\right)=-\alpha\left[\left(\left(\widehat{w}^{\varepsilon}\right)_{t}\right)_{(2)}(t)\right]^{2} .
$$

Passing to the limit, one obtains when $\varepsilon \rightarrow 0$ :

$$
\frac{d}{d t} E(t, \widehat{w})=-\alpha\left[\left(\widehat{w}_{t}\right)_{(2)}(t)\right]^{2}
$$

in the sense of distributions on $J$. Since $J$ was arbitrary, (54) holds on all compact subintervals of $[0, T]$. Then, let $t \in\left[0, \infty\left[\right.\right.$ be fixed, and let $\lim _{n \rightarrow \infty} t_{n}=t$. Let the sequence $v_{n}$ be defined by

$$
v_{n}=\frac{1}{2}\left\|\widehat{w}(t)-\widehat{w}\left(t_{n}\right)\right\|_{X}^{2}+\frac{1}{2}\left\|\widehat{w}_{t}(t)-\widehat{w}_{t}\left(t_{n}\right)\right\|_{Y}^{2}+\frac{\beta}{2}\left(\widehat{w}_{(2)}(t)-\widehat{w}_{(2)}\left(t_{n}\right)\right)^{2} .
$$

Thus, we have:

$$
v_{n}=\widehat{E}(t, \widehat{w})+\widehat{E}\left(t_{n}, \widehat{w}\right)-<\widehat{w}(t), \widehat{w}\left(t_{n}\right)>_{X}-<\widehat{w}_{t}(t), \widehat{w}_{t}\left(t_{n}\right)>_{Y}-\beta \widehat{w}_{(2)}(t) \widehat{w}_{(2)}\left(t_{n}\right) .
$$

Since $\widehat{w}, \widehat{w}_{t}$ are weakly continuous and $\widehat{E}$ is continuous in $t$, we have, passing to the limit in (56) :

$$
v_{n} \longrightarrow 0, \text { when } n \longrightarrow \infty
$$

Therefore, this implies that

$$
\left\|\widehat{w}(t)-\widehat{w}\left(t_{n}\right)\right\|_{X}^{2} \longrightarrow 0 \text { when } n \longrightarrow \infty
$$

and

$$
\left\|\widehat{w}_{t}(t)-\widehat{w}_{t}\left(t_{n}\right)\right\|_{Y}^{2} \longrightarrow 0 \text { when } n \longrightarrow \infty .
$$

Thus we get $\widehat{w} \in C([0, T] ; X)$ and $\widehat{w}_{t} \in C([0, T] ; Y)$.

In the next sections, the goal is to develop a numerical method for (1)-(4) in such a way that the decay of the Lyapunov function is preserved. The first step of this method is the discretization of the system in space to obtain the semi-discrete scheme, and then in time, in order to get the fully-discrete scheme.

\section{Semi-discrete Scheme}

\subsection{Piecewise Cubic Hermite Polynomials}

Our numerical work consist to construct an appropriate piecewise space of $C^{1}$-functions on $Z=[0,1]$. Assume that $Z$ is subdivided into $P$ intervals of the form $Z_{i}=\left[x_{i}, x_{i+1}\right], i=0, \ldots, P-1$ ie $Z=\cup_{i=0}^{P} Z_{i}$. In particular, if the subdivision is 
uniform, let us denote the step length by $h=1 / P$ and $Z_{i}=[i h,(i+1) h]$.

Let us find cubic polynomial functions $N_{j}^{i}, i=0,1, \ldots, P-1$ and $j=1,2,3,4$ that satisfy the following conditions :

$$
\begin{cases}N_{1}^{i}\left(x_{i}\right)=1, & N_{1 x}^{i}\left(x_{i}\right)=N_{1}^{i}\left(x_{i+1}\right)=N_{1 x}^{i}\left(x_{i+1}\right)=0, \\ N_{2 x}^{i}\left(x_{i}\right)=1, & N_{2}^{i}\left(x_{i}\right)=N_{2}^{i}\left(x_{i+1}\right)=N_{2 x}^{i}\left(x_{i+1}\right)=0, \\ N_{3}^{i}\left(x_{i+1}\right)=1, & N_{3}^{i}\left(x_{i}\right)=N_{3 x}^{i}\left(x_{i}\right)=N_{3 x}^{i}\left(x_{i+1}\right)=0, \\ N_{4 x}^{i}\left(x_{i+1}\right)=1, & N_{4}^{i}\left(x_{i}\right)=N_{4 x}^{i}\left(x_{i}\right)=N_{4}^{i}\left(x_{i+1}\right)=0, \quad \forall x \in Z_{i} .\end{cases}
$$

Let us use the following affine transformation:

$$
\theta_{i}=\frac{x-x_{i}}{x_{i+1}-x_{i}}, \quad \forall x \in\left[x_{i}, x_{i+1}\right],
$$

allowing us to manipulate all operations on $[0,1]$. The intermediate variables $\theta_{i}$ are called local coordinates. Then under these coordinates, the unknown functions $\widetilde{N}_{j}(\theta)$ must satisfy the following boundary conditions:

$$
\begin{cases}\widetilde{N}_{1}(0)=1, & \widetilde{N}_{1 \theta}(0)=\widetilde{N}_{1}(1)=\widetilde{N}_{1 \theta}(1)=0 \\ \widetilde{N}_{2 \theta}(0)=1, & \widetilde{N}_{2}(0)=\widetilde{N}_{2}(1)=\widetilde{N}_{2 \theta}(1)=0 \\ \widetilde{N}_{3}(1)=1, & \widetilde{N}_{3}(0)=\widetilde{N}_{3 \theta}(0)=\widetilde{N}_{3 \theta}(1)=0 \\ \widetilde{N}_{4 \theta}(1)=1, & \widetilde{N}_{4}(0)=\widetilde{N}_{4 \theta}(0)=\widetilde{N}_{4}(1)=0\end{cases}
$$

Let us find explicit expressions of the functions $\widetilde{N}_{j}(\theta)$. For all $\theta \in[0,1]$, for all $j=1,2,3,4$, the functions $\widetilde{N}_{j}(\theta)$ are polynomials of degree 3 , therefore of the form $\widetilde{N}_{j}(\theta)=a \theta^{3}+b \theta^{2}+c \theta+d$. We remark that 1 is a double root of $N_{1}$. Then $\widetilde{N}_{1}(\theta)=(\theta-1)^{2}(a \theta+b)$. Using the two remaining conditions, we find $a=2$ and $b=1$. Hence we get

$$
\widetilde{N}_{1}(\theta)=(\theta-1)^{2}(2 \theta+1) \text {. }
$$

Analogously, we find

$$
\widetilde{N}_{2}(\theta)=\theta(\theta-1)^{2}, \widetilde{N}_{3}(\theta)=\theta^{2}(3-2 \theta), \widetilde{N}_{4}(\theta)=\theta^{2}(\theta-1), \forall \theta \in[0,1] .
$$

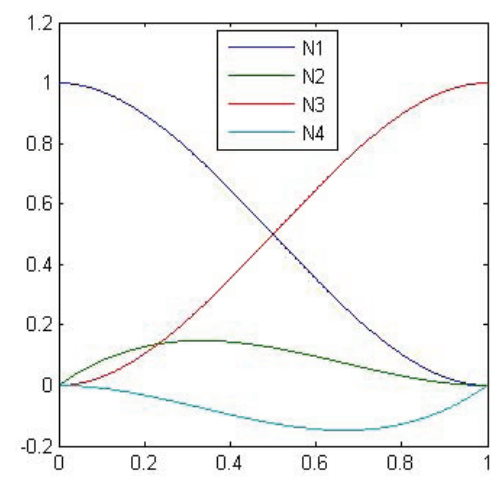

Figure 2. Hermitian Polynomial functions

By extension by 0 on $Z-Z_{i}$, for all $i=0, \ldots, P-1$, we define the Hermitian functions on $Z$ :

$$
\left\{\begin{array}{l}
N_{j}^{i}(x)=\left.N_{j}\left(\theta_{i}\right)\right|_{\theta_{i}=} \frac{x-x_{i}}{h}, j=1,3 \\
N_{j}^{i}(x)=\left.h N_{j}\left(\theta_{i}\right)\right|_{\theta_{i}=\frac{x-x_{i}}{h}}, j=2,4 \\
N_{j}^{i}=\left.0\right|_{Z-Z_{i}}, i=0, \ldots, P-1, j=1,2,3,4 .
\end{array}\right.
$$


By concatenating the Hermitian functions obtained above, we can obtain the basic functions on the different supports as follows:

with the support $Z_{0}, \phi_{1}^{0}(x)=N_{1}^{0}(x), \phi_{2}^{0}(x)=N_{2}^{0}(x)$;

with the support $Z_{i-1}, \phi_{1}^{i}(x)=N_{3}^{i-1}(x), \phi_{2}^{i}(x)=N_{4}^{i-1}(x)$;

with the support $Z_{i}, \phi_{1}^{i}(x)=N_{1}^{i}(x), \phi_{2}^{i}(x)=N_{2}^{i}(x)$

and with the support $Z_{P}, \phi_{1}^{P}(x)=N_{3}^{P-1}(x), \phi_{2}^{P}(x)=N_{4}^{P-1}(x)$.

The set $\bar{B}=\left\{\phi_{l}^{k}, k=0, \ldots, P, l=1,2\right\}$ forms a basis that generates a subspace of $V$ of dimension $2 P+2$ denoted by $\widetilde{V}^{h}$.

With the separation of variables, the approximate solution $w_{h} \in \widetilde{V}^{h}$ which we seek can be written as follows:

$$
w_{h}(x, t)=\sum_{j=0}^{N}\left[w_{h}^{j}(t) \phi_{1}^{j}(x)+\widetilde{w}_{h}^{j}(t) \phi_{2}^{j}(x)\right] .
$$

Thus, since $w_{h}^{0}=\widetilde{w}_{h}^{0}=0$, the N-dimensional space is as follows:

$$
V^{h}=\operatorname{Span}\left\{\phi_{1}^{1}, \phi_{2}^{1}, \ldots, \phi_{1}^{P}, \phi_{2}^{P}\right\}
$$

\subsection{Semi-discrete Scheme: Space Discretization}

Since rounding errors are cumulative because of the high partial derivative terms (the spatial derivatives being of order 4) in the equation of the beam (1), we propose a finite element scheme semi-discretized in space. In fact, a variational approach allows us to reduce the degree of the derivatives by the integration by parts.

Let $\phi_{j}, j=1, \ldots, N$ be fixed basis for $V^{h}$. The semi-discrete solution $w_{h} \in C^{2}\left(\left[0, \infty\left[, V^{h}\right)\right.\right.$ is defined as the solution of the finite element method:

$$
\int_{0}^{1}\left(w_{h}\right)_{t t} \phi_{j} \mathrm{~d} x+\int_{0}^{1}\left(w_{h}\right)_{x x}\left(\phi_{j}\right)_{x x} \mathrm{~d} x+\beta\left(w_{h}\right)_{x}(1)\left(\phi_{j}\right)_{x}(1)+\alpha\left(w_{h}\right)_{t x}(1)\left(\phi_{j}\right)_{x}(1)=0
$$

for all $j=1, \ldots, N$ and $t>0$, which solves the initial conditions

$$
\begin{gathered}
w_{h}(., 0)=w_{h, 0} \in V^{h} \\
\left(w_{h}\right)_{t}(., 0)=v_{h, 0} \in V^{h} .
\end{gathered}
$$

Equation (62) is a second order ODE-system in time. By separation of variables, its solution can be written in the following form:

$$
\left(w_{h}\right)(x, t)=\sum_{i=1}^{N} W_{i}(t) \phi_{i}(x)
$$

where $W$ is a vector representation of the function $w_{h}$ defined as follows:

$$
W(t)=\left[\begin{array}{lllllll}
W_{1}(t) & W_{2}(t) & \ldots & W_{P}(t) & W_{1 x}(t) & \ldots & W_{P x}(t)
\end{array}\right]^{T} .
$$

Equation (62) is equivalent to the following equation:

$$
M W_{t t}+S W_{t}+K W=0 .
$$

$M$ is the mass matrix and $K$ is the rigidity matrix. The corresponding matrices $M, S$ and $K$ are given by:

$$
\begin{gathered}
M_{i j}=\int_{0}^{1} \phi_{i} \phi_{j} d x, \quad S_{i j}=\alpha\left(\phi_{i}\right)_{x}(1)\left(\phi_{j}\right)_{x}(1) \quad \forall i, j=1, \ldots, N, \\
K_{i j}=\int_{0}^{1}\left(\phi_{i}\right)_{x x}\left(\phi_{j}\right)_{x x} d x+\beta\left(\phi_{i}\right)_{x}(1)\left(\phi_{j}\right)_{x}(1) \quad \forall i, j=1, \ldots, N .
\end{gathered}
$$

The matrix $K$ is symmetric, defined and positive because $\beta>0$ and therefore $K$ is invertible. Since the matrix $M$ is also symmetric, defined and positive, this implies the existence and the uniqueness of the solution of problem (62)-(64). Note that $M$ and $K$ are tridiagonal matrices by blocks while $S$ is diagonal. The calculation of elements of $S$ is trivial because all the elements of $S$ are zero except one nonzero element $S_{N, N}=\alpha$ with $N=2 P$. 


\section{Values of elements of matrices $M$ and $K$}

For $i=1, \ldots, N$

$$
\begin{aligned}
& M_{2 i-3,2 i-3}=\int_{x_{i-1}}^{x_{i}}\left(\phi_{1}^{2 i-3}\right)^{2} \mathrm{~d} x=\frac{13}{35} h, \\
& M_{2 i-2,2 i-2}=\int_{x_{i-1}}^{x_{i}}\left(\phi_{2}^{2 i-2}\right)^{2} \mathrm{~d} x=\frac{1}{105} h^{3}, \\
& M_{2 i-1,2 i-1}=\int_{x_{i}}^{x_{i+1}}\left(\phi_{1}^{2 i-1}\right)^{2} \mathrm{~d} x=\frac{13}{35} h, \\
& M_{2 i, 2 i}=\int_{x_{i}}^{x_{i+1}}\left(\phi_{2}^{2 i}\right)^{2} \mathrm{~d} x=\frac{1}{105} h^{3}, \\
& M_{2 i-1,2 i-3}=\int_{x_{i}}^{x_{i+1}}\left(\phi_{1}^{2 i-1}\right)\left(\phi_{1}^{2 i-3}\right) \mathrm{d} x=\frac{9}{70} h, \\
& M_{2 i-3,2 i-2}=\int_{x_{i-1}}^{x_{i}}\left(\phi_{1}^{2 i-3}\right)\left(\phi_{2}^{2 i-2}\right) \mathrm{d} x=\frac{11}{210} h^{2}, \\
& M_{2 i-1,2 i}=\int_{x_{i}}^{x_{i+1}}\left(\phi_{1}^{2 i-1}\right)\left(\phi_{2}^{2 i}\right) \mathrm{d} x=-\frac{11}{210} h^{2}, \\
& M_{2 i-3,2 i}=\int_{x_{i}}^{x_{i+1}}\left(\phi_{1}^{2 i-3}\right)\left(\phi_{2}^{2 i}\right) \mathrm{d} x=-\frac{13}{420} h^{2}, \\
& M_{2 i-1,2 i-2}=\int_{x_{i}}^{x_{i+1}}\left(\phi_{1}^{2 i-1}\right)\left(\phi_{2}^{2 i-2}\right) \mathrm{d} x=\frac{13}{420} h^{2}, \\
& M_{2 i-1,2 i-2}=\int_{x_{i}}^{x_{i+1}}\left(\phi_{1}^{2 i-1}\right)\left(\phi_{2}^{2 i-2}\right) \mathrm{d} x=\frac{13}{420} h^{2}, \\
& M_{2 i, 2 i-2}=\int_{x_{i}}^{x_{i+1}}\left(\phi_{2}^{2 i}\right)\left(\phi_{2}^{2 i-2}\right) \mathrm{d} x=\frac{1}{140} h^{3} . \\
& K_{2 i-3,2 i-3}=\int_{x_{i-1}}^{x_{i}}\left(\left(\phi_{1}^{2 i-3}\right)^{\prime \prime}\right)^{2} \mathrm{~d} x=\frac{12}{h^{3}}, \\
& K_{2 i-2,2 i-2}=\int_{x_{i-1}}^{x_{i}}\left(\left(\phi_{2}^{2 i-2}\right)^{\prime \prime}\right)^{2} \mathrm{~d} x=\frac{4}{h}, \\
& K_{2 i-1,2 i-1}=\int_{x_{i}}^{x_{i+1}}\left(\left(\phi_{1}^{2 i-1}\right)^{\prime \prime}\right)^{2} \mathrm{~d} x=\frac{12}{h^{3}}, \\
& K_{2 i, 2 i}=\int_{x_{i}}^{x_{i+1}}\left(\left(\phi_{2}^{2 i}\right)^{\prime \prime}\right)^{2} \mathrm{~d} x=\frac{4}{h}, \\
& K_{2 i-1,2 i-3}=\int_{x_{i}}^{x_{i+1}}\left(\phi_{1}^{2 i-1}\right)^{\prime \prime}\left(\phi_{1}^{2 i-3}\right)^{\prime \prime} \mathrm{d} x=-\frac{12}{h^{3}}, \\
& K_{2 i-3,2 i-2}=\int_{x_{i-1}}^{x_{i}}\left(\phi_{1}^{2 i-3}\right)^{\prime \prime}\left(\phi_{2}^{2 i-2}\right)^{\prime \prime} \mathrm{d} x=\frac{6}{h^{2}}, \\
& K_{2 i-1,2 i}=\int_{x_{i}}^{x_{i+1}}\left(\phi_{1}^{2 i-1}\right)^{\prime \prime}\left(\phi_{2}^{2 i}\right)^{\prime \prime} \mathrm{d} x=-\frac{6}{h^{2}}, \\
& K_{2 i-3,2 i}=\int_{x_{i}}^{x_{i+1}}\left(\phi_{1}^{2 i-3}\right)^{\prime \prime}\left(\phi_{2}^{2 i}\right)^{\prime \prime} \mathrm{d} x=\frac{6}{h^{2}}, \\
& K_{2 i-1,2 i-2}=\int_{x_{i}}^{x_{i+1}}\left(\phi_{1}^{2 i-1}\right)^{\prime \prime}\left(\phi_{2}^{2 i-2}\right)^{\prime \prime} \mathrm{d} x=-\frac{6}{h^{2}} \text {, } \\
& K_{2 i, 2 i-2}=\int_{x_{i}}^{x_{i+1}}\left(\phi_{2}^{2 i}\right)^{\prime \prime}\left(\phi_{2}^{2 i-2}\right)^{\prime \prime} \mathrm{d} x=\frac{2}{h} .
\end{aligned}
$$




\subsection{Dissipativity of the Semi-discrete Scheme}

In order to show that the scheme given by (62)-(64) is dissipative, first a time dependent energy functional $E$ for a trajectory $w \in C^{2}([0, \infty[, V)$ is defined as analogous of the Lyapunov functional (13).

Theorem 12 Let $w_{h} \in C\left(\left[0, \infty\left[; V^{h}\right)\right.\right.$ solution of (62)-(64). Then we get:

$$
\forall t>0, \frac{d}{d t} E\left(t, w_{h}\right)=-\alpha\left[\left(w_{h}\right)_{t x}(1)\right]^{2} \leq 0 .
$$

Proof. Derive for all $t>0, E\left(t, w_{h}\right)$. We have:

$$
\forall t>0, \frac{d}{d t} E\left(t, w_{h}\right)=\int_{0}^{1}\left(w_{h}\right)_{x x t}\left(w_{h}\right)_{x x} \mathrm{~d} x+\int_{0}^{1}\left(w_{h}\right)_{t t}\left(w_{h}\right)_{t} \mathrm{~d} x+\beta\left(w_{h}\right)_{t x}(1)\left(w_{h}\right)_{x}(1) .
$$

Using (62) with the test function $\phi_{h}=\left(w_{h}\right)_{t}$, we obtain:

$$
\forall t>0, \quad \int_{0}^{1}\left(w_{h}\right)_{t t}\left(w_{h}\right)_{t} \mathrm{~d} x+\int_{0}^{1}\left(w_{h}\right)_{x x}\left(\left(w_{h}\right)_{x x t} \mathrm{~d} x+\beta\left(w_{h}\right)_{x}(1)\left(\left(w_{h}\right)_{t x}(1)+\alpha\left(w_{h}\right)_{t x}(1)\left(w_{h}\right)_{t x}(1)=0 .\right.\right.
$$

Then, we get the result (67).

Remark 3 Note that the property of dissipativity theorem of the norm was written independently of the basis $\phi_{j}, j=1, \ldots, N$ and can be applied to any choice of the subspace $V^{h} \subset V$.

\subsection{A-priori Error Estimates}

In this subsection, the a-priori error estimates for the semi-discrete solution approximation (62) are obtained. We will use a common method used in (Choo, Chung \& Kannan, 2002) to obtain error estimates. Of course, we will adapt the method used in this article to our problem. The projection of weak solution $w$ to $V^{h}$ on $H^{2}(0,1)$ denoted by $\bar{w}$ is defined as follows:

$$
\forall x \in(0,1), \forall t>0, \bar{w}(x, t)=\sum_{j=1}^{N} w\left(t, x_{j}\right) \phi_{1}^{j}(x)+\sum_{j=1}^{N} w_{x}\left(t, x_{j}\right) \phi_{2}^{j}(x) .
$$

We set $G=\left\{w \in H^{4}(0,1), w(0)=w_{x}(0)=0\right\}$. Assume for later that:

$$
\begin{gathered}
w \in C([0, T] ; G), \\
w_{t} \in L^{2}([0, T] ; G), \\
w_{t t} \in L^{2}([0, T] ; V) .
\end{gathered}
$$

Then, from the lemma 2.1 of (Choo, Chung \& Kannan, 2002), we have the following estimations almost every in $t$ :

$$
\begin{gathered}
\|w-\bar{w}\|_{H^{2}(0,1)} \leq C h^{2}\|w\|_{H^{4}(0,1)}, \\
\left\|w_{t}-\bar{w}_{t}\right\|_{H^{2}(0,1)} \leq C h^{2}\left\|w_{t}\right\|_{H^{4}(0,1)}, \\
\left\|w_{t t}-\bar{w}_{t t}\right\|_{L^{2}(0,1)} \leq C h^{2}\left\|w_{t t}\right\|_{H^{2}(0,1)} .
\end{gathered}
$$

Now, we give an important result for the convergence of the semi-discrete scheme:

Theorem 13 Let $V^{h}$ the space of cubic Hermite polynomials. Assume the expressions (70)-(72). The following error estimate holds for $w_{h} \in C^{2}\left([0, T], V^{h}\right)$ solving (62) is given by:

$\forall t \in[0, T],\left[E\left(t, w_{h}-w\right)\right]^{1 / 2} \leq C\left(E\left(0, w_{h}(0)-w(0)\right)^{1 / 2}+h^{2}\left(\|w\|_{C\left([0, T], H^{4}(0,1)\right)}^{2}+\left\|w_{t}\right\|_{L^{2}\left([0, T], H^{4}(0,1)\right)}^{2}+\left\|w_{t t}\right\|_{L^{2}\left([0, T], H^{2}(0,1)\right)}^{2}\right)\right)$.

Furthermore, if $w_{h 0}$ and $v_{h 0}$ are respectively Hermite interpolations of $w_{0}$ and of $v_{0}$, then there exists a positive constant C such that:

$$
\left[E\left(t, w_{h}-w\right)\right]^{1 / 2} \leq C h^{2}\left(\|w\|_{C\left([0, T], H^{4}(0,1)\right)}+\left\|w_{t}\right\|_{L^{2}\left([0, T], H^{4}(0,1)\right)}+\left\|w_{t t}\right\|_{L^{2}\left([0, T], H^{2}(0,1)\right)}\right)
$$


Proof. The error of semi-discrete solution $w_{h}$ is defined as $e_{h}=w_{h}-\bar{w}$. We remark that $e_{h}$ is an element of $V^{h}$. Then, substituting $w_{h}=e_{h}+\bar{w}$ in (62), we get:

$$
\begin{aligned}
& \int_{0}^{1}\left(e_{h}\right)_{t t} \phi \mathrm{d} x+\int_{0}^{1}\left(e_{h}\right)_{x x} \phi_{x x} \mathrm{~d} x+\beta\left(e_{h}\right)_{x}(1) \phi_{x}(1)+\alpha\left(e_{h}\right)_{t x}(1) \phi_{x}(1) \\
= & -\int_{0}^{1}(\bar{w})_{t t} \phi \mathrm{d} x-\int_{0}^{1}(\bar{w})_{x x} \phi_{x x} \mathrm{~d} x-\beta(\bar{w})_{x}(1) \phi_{x}(1)-\alpha(\bar{w})_{t x}(1) \phi_{x}(1)
\end{aligned}
$$

for all $\phi \in V^{h}$. Furthermore, $\bar{w}$ is the projection of weak solution $w$ on discret espace $V^{h}$.

Therefore using again (62), we have the following equation:

$$
\int_{0}^{1}\left(e_{h}\right)_{t t} \phi \mathrm{d} x+\int_{0}^{1}\left(e_{h}\right)_{x x} \phi_{x x} \mathrm{~d} x+\beta\left(e_{h}\right)_{x}(1) \phi_{x}(1)+\alpha\left(e_{h}\right)_{t x}(1) \phi_{x}(1)=\int_{0}^{1}(w-\bar{w})_{t t} \mathrm{~d} x+\int_{0}^{1}(w-\bar{w})_{x x} \phi_{x x} \mathrm{~d} x
$$

for all $\phi \in V^{h}$. Taking now $\phi=\left(e_{h}\right)_{t} \in V^{h}$. Thereby, (78) becomes :

$$
\begin{aligned}
& \int_{0}^{1}\left(e_{h}\right)_{t t}\left(e_{h}\right)_{t} \mathrm{~d} x+\int_{0}^{1}\left(e_{h}\right)_{x x}\left(e_{h}\right)_{t x x} \mathrm{~d} x+\beta\left(e_{h}\right)_{x}(1)\left(e_{h}\right)_{t x}(1)+\alpha\left(e_{h}\right)_{t x}(1)\left(e_{h}\right)_{t x}(1) \\
= & \int_{0}^{1}(w-\bar{w})_{t t}\left(e_{h}\right)_{t} \mathrm{~d} x+\int_{0}^{1}(w-\bar{w})_{x x}\left(e_{h}\right)_{t x x} \mathrm{~d} x, \quad \forall t \in[0, T] .
\end{aligned}
$$

For all $t \in[0, T]$,

$$
\frac{1}{2} \frac{d}{d t}\left[\int_{0}^{1}\left(e_{h}\right)_{t t}^{2} \mathrm{~d} x+\int_{0}^{1}\left(e_{h}\right)_{x x}^{2} \mathrm{~d} x+\beta\left(\left(e_{h}\right)_{x}(1)\right)^{2}\right]=\int_{0}^{1}(w-\bar{w})_{t t}\left(e_{h}\right)_{t} \mathrm{~d} x+\int_{0}^{1}(w-\bar{w})_{x x}\left(e_{h}\right)_{t x x} \mathrm{~d} x-\alpha\left(e_{h}\right)_{t x}(1)\left(e_{h}\right)_{t x}(1)
$$

Thus we get:

$$
\frac{1}{2} \frac{d}{d t} E\left(t, e_{h}\right)=\int_{0}^{1}(w-\bar{w})_{t t}\left(e_{h}\right)_{t} \mathrm{~d} x+\int_{0}^{1}(w-\bar{w})_{x x}\left(e_{h}\right)_{t x x} \mathrm{~d} x-\alpha\left(e_{h}\right)_{t x}(1)\left(e_{h}\right)_{t x}(1), \quad \forall t \in[0, T] .
$$

This implies that:

$$
\frac{1}{2} \frac{d}{d t} E\left(t, e_{h}\right) \leq \int_{0}^{1}(w-\bar{w})_{t t}\left(e_{h}\right)_{t} \mathrm{~d} x+\int_{0}^{1}(w-\bar{w})_{x x}\left(e_{h}\right)_{t x x} \mathrm{~d} x, \quad \forall t \in[0, T]
$$

Or again :

$$
\frac{d}{d t} E\left(t, e_{h}\right) \leq 2 \int_{0}^{1}(w-\bar{w})_{t t}\left(e_{h}\right)_{t} \mathrm{~d} x+2 \int_{0}^{1}(w-\bar{w})_{x x}\left(e_{h}\right)_{t x x} \mathrm{~d} x, \quad \forall t \in[0, T] .
$$

By integrating (81) in the time direction ie on $t \in[0, T]$, one obtains:

$$
E\left(t, e_{h}\right) \leq E\left(0, e_{h}(0)\right)+2 \int_{0}^{t} \int_{0}^{1}(w-\bar{w})_{t t}\left(e_{h}\right)_{t} \mathrm{~d} x \mathrm{~d} \tau+2 \int_{0}^{t} \int_{0}^{1}(w-\bar{w})_{x x}\left(e_{h}\right)_{t x x} \mathrm{~d} x \mathrm{~d} \tau .
$$

By one integration by parts $\int_{0}^{t}(w-\bar{w})_{x x}\left(e_{h}\right)_{t x x} \mathrm{~d} \tau,(82)$ becomes finally:

$$
\begin{aligned}
\forall t \in[0, T], E\left(t, e_{h}\right) \leq E\left(0, e_{h}(0)\right) & +2 \int_{0}^{t} \int_{0}^{1}\left(w_{t t}(\tau, x)-\bar{w}_{t t}(\tau, x)\right)\left(e_{h}\right)_{t}(\tau, x) \mathrm{d} x \mathrm{~d} \tau \\
& -2 \int_{0}^{t} \int_{0}^{1}\left(w_{t x x}(\tau, x)-\bar{w}_{t x x}(\tau, x)\right)\left(e_{h}\right)_{x x}(\tau, x) \mathrm{d} x \mathrm{~d} \tau \\
& +2 \int_{0}^{1}\left(w_{x x}(t, x)-\bar{w}_{x x}(t, x)\right)\left(e_{h}\right)_{x x}(t, x) \mathrm{d} x \\
& +2 \int_{0}^{1}\left(w_{x x}(0, x)-\bar{w}_{x x}(0, x)\right)\left(e_{h}\right)_{x x}(0, x) \mathrm{d} x
\end{aligned}
$$

Using Cauchy Schwarz's inequality to (83) yields

$$
\begin{aligned}
E\left(t, e_{h}\right) \leq & E\left(0, e_{h}(0)\right)+C_{1}\left[\left\|w_{t t}-\bar{w}_{t t}\right\|_{L^{2}\left([0, T], L^{2}(0,1)\right)}^{2}+\int_{0}^{t}\left\|\left(e_{h}\right)_{t}(\tau, .)\right\|_{L^{2}(0,1)}^{2} \mathrm{~d} \tau\right] \\
& +C_{2}\left[\left\|w_{x x}(t, .)-\bar{w}_{x x}(t, .)\right\|_{L^{2}(0,1)}^{2}+\left\|\left(e_{h}\right)_{x x}(t, .)\right\|_{L^{2}(0,1)}^{2}\right] \\
& +C_{3}\left[\left\|w_{x x}(0, .)-\bar{w}_{x x}(0, .)\right\|_{L^{2}(0,1)}^{2}+\left\|\left(e_{h}\right)_{x x}(0, .)\right\|_{L^{2}(0,1)}^{2}\right] \\
& +C_{4}\left[\left\|w_{t}-\bar{w}_{t}\right\|_{L^{2}\left([0, T], H^{2}(0,1)\right)}^{2}+\int_{0}^{t}\left\|\left(e_{h}\right)_{x x}(\tau, .)\right\|_{L^{2}(0,1)}^{2} \mathrm{~d} \tau\right],
\end{aligned}
$$


where $C_{1}, C_{2}, C_{3}$ and $C_{4}$ are positive constants.

Using estimations (73)-(75) and Gronwall's inequality, we obtain (76). Finally, using the triangle inequality, we get the result (77).

Remark 4 The order of convergence for the discretized scheme in space is 2 .

\section{Fully-discrete Scheme}

In this section, in order to obtain a fully discretized scheme, we discretize in time of semi-discretized system (62) in such a way that the dissipation of energy is preserved. To achieve this goal, the system (62) is written as a system of ordinary differential equations of first order. Then, the Crank-Nicolson scheme obtained is used to demonstrate dissipativity of the numerical scheme. Finally, the a-priori estimates are obtained.

\subsection{Crank-Nicolson Scheme}

Let $L$ be positive integer. Here, the interval $[0, T]$ is discretized into $L$ equidistant subintervals. Let $k=T / L$ denotes the size of time discretization and $t_{n}=n k$ where $n=0,1, \ldots, L$ represent the nodes of the discretization. In order to rewrite the semi-discretized scheme (62) as a differential equation of the first order, introduce the element $v_{h}=\left(w_{h}\right)_{t}$. Furthermore, let $V=W_{t}=\left[\begin{array}{lllll}V_{1} & V_{2} & \ldots & V_{N}\end{array}\right]^{T}$ be its vector representation in the basis $\left\{\phi_{j}\right\}_{j=1}^{N}$ of Hermitian cubic polynomial space. Note that the solution $w_{h}$ of semi-discretized scheme (62) becomes for the full discrete scheme a vector of the form $y_{h}=\left[\begin{array}{ll}w_{h} & v_{h}\end{array}\right]^{T}$. Furthermore, similar to (13), the natural norm of $y_{h}=y_{h}(t)$ is defined as follows:

$$
\left\|y_{h}\right\|^{2}=\frac{1}{2} \int_{0}^{1}\left[\left(w_{h}\right)_{x x}^{2}+v_{h}^{2}\right] \mathrm{d} x+\frac{\beta}{2}\left(\left(w_{h}\right)_{x}(1)\right)^{2} .
$$

Let now $y^{n}=\left[\begin{array}{ll}w^{n} & v^{n}\end{array}\right]$ be the approximate solution of $y_{h}$ in time $t=t_{n}$. Let again $W^{n}=W\left(x, t_{n}\right)$ and $V^{n}=V\left(x, t_{n}\right)$ the vector representations in basis $\left\{\phi_{j}\right\}_{j=1}^{N}$ respectively of $w^{n}$ and $v^{n}$

For the time discretization of (62), the Crank-Nicolson scheme

$$
\frac{w^{n+1}-w^{n}}{k}=\frac{v^{n+1}+v^{n}}{2}
$$

is used. Then we get:

$$
\int_{0}^{1} \frac{v^{n+1}-v^{n}}{k} \phi_{h} \mathrm{~d} x+\int_{0}^{1} \frac{w_{x x}^{n+1}+w_{x x}^{n}}{2}\left(\phi_{h}\right)_{x x} \mathrm{~d} x+\beta \frac{w_{x}^{n+1}(1)+w_{x}^{n}(1)}{2}\left(\phi_{h}\right)_{x}(1)+\alpha \frac{v_{x}^{n+1}(1)+v_{x}^{n}(1)}{2}\left(\phi_{h}\right)_{x}(1)=0
$$

for all $\phi_{h} \in V^{h}$.

Furthermore, the vector equation (66) becomes:

$$
\frac{M V^{n+1}-M V^{n}}{k}+\frac{S V^{n+1}+S V^{n}}{2}+\frac{K W^{n+1}+K W^{n}}{2}=0
$$

which is equivalent to:

$$
\left(\frac{M}{k}+\frac{S}{2}\right) V^{n+1}+\frac{K}{2} W^{n+1}=-\frac{K}{2} W^{n}+\left(\frac{M}{k}-\frac{S}{2}\right) V^{n}
$$

(84) and (87) give us the following system of equation: $P Y^{n+1}=Q Y^{n}$ where $P$ and $Q$ are block matrices defined as follows:

and the vector $Y^{n}=\left[\begin{array}{ll}W^{n} & V^{n}\end{array}\right]$.

$$
\begin{aligned}
& P=\left(\begin{array}{cc}
\frac{I}{k} & -\frac{I}{2} \\
\frac{K}{2} & -\frac{M}{k}+\frac{S}{2}
\end{array}\right) \\
& Q=\left(\begin{array}{cc}
\frac{I}{k} & \frac{I}{2} \\
-\frac{K}{2} & \frac{M}{k}-\frac{S}{2}
\end{array}\right)
\end{aligned}
$$

\subsection{Dissipativity of Numerical Scheme}

Now, we show that the fully discrete scheme (84) and (86) dissipates the norm (energy) in time. 
Theorem 14 For all $n=0,1, \ldots, L, L$ and $k$ the positive integers, we get

$$
\left\|y^{n+1}\right\|^{2}-\left\|y^{n}\right\|^{2}=-\alpha \frac{\left(w_{x}^{n+1}(1)-w_{x}^{n}(1)\right)^{2}}{k} \leq 0 .
$$

Proof. We have

$$
\begin{aligned}
\left\|y^{n+1}\right\|^{2}-\left\|y^{n}\right\|^{2} & =\frac{1}{2} \int_{0}^{1}\left(w_{x x}^{n+1}\right)^{2} \mathrm{~d} x-\frac{1}{2} \int_{0}^{1}\left(w_{x x}^{n}\right)^{2} \mathrm{~d} x+\frac{1}{2} \int_{0}^{1}\left(v^{n+1}\right)^{2} \mathrm{~d} x \\
& -\frac{1}{2} \int_{0}^{1}\left(v^{n}\right)^{2} \mathrm{~d} x+\frac{\beta}{2}\left(w_{x}^{n+1}(1)\right)^{2}-\frac{\beta}{2}\left(w_{x}^{n}(1)\right)^{2} .
\end{aligned}
$$

Multiplying (84) by $v^{n+1}-v^{n}$ and integrating over [0,1], we obtain:

$$
\int_{0}^{1} \frac{w^{n+1}-w^{n}}{k}\left(v^{n+1}-v^{n}\right) \mathrm{d} x=\int_{0}^{1} \frac{\left(v^{n+1}\right)^{2}-\left(v^{n}\right)^{2}}{2} \mathrm{~d} x .
$$

Taking $\phi_{h}=w^{n+1} \in V^{h}$ in (85) yields:

$$
\begin{aligned}
\frac{1}{2} \int_{0}^{1}\left(w_{x x}^{n+1}\right)^{2} \mathrm{~d} x=- & \frac{1}{2} \int_{0}^{1} w_{x x}^{n+1} w_{x x}^{n} \mathrm{~d} x-\int_{0}^{1} \frac{v^{n+1}-v^{n}}{k} w^{n+1} \mathrm{~d} x \\
& -\beta \frac{w_{x}^{n+1}(1)+w_{x}^{n}(1)}{2} w_{x}^{n+1}(1)-\alpha \frac{v_{x}^{n+1}(1)+v_{x}^{n}(1)}{2} w_{x}^{n+1}(1) .
\end{aligned}
$$

Taking now $\phi_{h}=w^{n} \in V^{h}$ in (85). Hence we get:

$$
\begin{aligned}
\frac{1}{2} \int_{0}^{1}\left(w_{x x}^{n}\right)^{2} \mathrm{~d} x= & -\frac{1}{2} \int_{0}^{1} w_{x x}^{n+1} w_{x x}^{n} \mathrm{~d} x-\int_{0}^{1} \frac{v^{n+1}-v^{n}}{k} w^{n} \mathrm{~d} x \\
& -\beta \frac{w_{x}^{n+1}(1)+w_{x}^{n}(1)}{2} w_{x}^{n}(1)-\alpha \frac{v_{x}^{n+1}(1)+v_{x}^{n}(1)}{2} w_{x}^{n}(1) .
\end{aligned}
$$

Thus, using (89)-(91) and again (84), we obtain the result (88).

Remark 5 The norm dissipates in time : $\left\|y^{n+1}\right\|^{2} \leq\left\|y^{n}\right\|^{2}$. This decay of the norm when $k \longrightarrow 0$ corresponds to the decay of the norm (14) in the continuous case and with the norm (67) in the semi-discrete case. However, if the beam is not controlled ie when $\alpha=\beta=0$ then $\left\|y^{n+1}\right\|=\left\|y^{n}\right\|$, therefore the norm $\left\|y^{n}\right\|$ is constant for all $n=0,1, \ldots, L$ where $L$ is a positive integer. Also, notice that the Crank Nicolson scheme (84), the expression(86) and the norm dissipation property from Theorem 14 of the norm were written independently of the basis $\left\{\phi_{j}\right\}$. Therefore, this property of dissipativity can be applied to any choice of the subspace $V^{h} \subset V$.

\subsection{A-priori Error Estimates}

Assume that $w \in H^{4}(0, T ; V)$.

Let $\bar{w} \in V^{h}$ be defined as the projection of the weak solution $w$ on $V^{h}$ such that $a_{1}\left(\bar{w}(t), \phi_{h}\right)=a_{1}\left(w(t), \phi_{h}\right)$ for all $\phi_{h} \in V^{h}$, for all $t \in[0, T]$. If $w \in H^{4}([0, T] ; V)$ then $\bar{w} \in H^{4}([0, T] ; V)$ since $\bar{w}$ is bounded in $V$. Moreover, let $w^{e}:=w-\bar{w}$ denote the error of the projection. Assume also that:

$$
w \in H^{2}([0, T] ; G), \quad w_{t} \in L^{2}([0, T] ; G), \quad w_{t t} \in H^{2}([0, T] ; V) .
$$

Then due to ( Strang \& Fix, 1973), we have the following estimations:

$$
\begin{gathered}
\|w-\bar{w}\|_{H^{2}(0,1)} \leq C h^{2}\|w\|_{H^{4}(0,1)}, \\
\left\|w_{t}-\bar{w}_{t}\right\|_{H^{2}(0,1)} \leq C h^{2}\left\|w_{t}\right\|_{H^{4}(0,1)}, \\
\left\|w_{t t}-\bar{w}_{t t}\right\|_{H^{2}(0,1)} \leq C h^{2}\left\|w_{t t}\right\|_{H^{4}(0,1)} .
\end{gathered}
$$

Let $y\left(t_{n}\right)=\left[\begin{array}{lll}w\left(t_{n}\right) & w_{t}\left(t_{n}\right)\end{array}\right]^{T}$ denotes the weak solution of (18) at time $t=t_{n}$. This approximation is defined by $y^{n}=$ $\left[\begin{array}{cc}w^{n} & v^{n}\end{array}\right]^{T}$, the $n$-th iteration of the fully-discrete scheme of Crank Nicolson. Thus, the approximation error is defined by $y_{e}^{n}=\left[\begin{array}{ll}e_{1}^{n} & e_{2}^{n}\end{array}\right]^{T}$ with $e_{1}^{n}=w^{n}-\bar{w}\left(t_{n}\right), e_{2}^{n}=v^{n}-\bar{w}_{t}\left(t_{n}\right)$ for all $n=0,1, \ldots, L$. 
Therefore, the second order error estimate both in space and time of the fully discrete scheme is obtained in the following Theorem.

Theorem 15 Assume $w \in H^{2}([0, T] ; G) \cap H^{4}([0, T] ; V)$. Also, take $n=1, \ldots, L$. Then the estimate is translated as follows:

$$
\left\|y^{n}-y\left(t_{n}\right)\right\| \leq M\left[\left\|y_{e}^{0}\right\|+h^{2}\|w\|_{H^{2}\left([0, T] ; H^{4}(0,1)\right)}+k^{2}\left(\left\|w_{t t}\right\|_{L^{2}\left([0, T] ; H^{4}(0,1)\right)}+\left\|w_{t t}\right\|_{H^{2}\left([0, T] ; H^{2}(0,1)\right)}\right)\right]
$$

where $M$ is a positive constant.

Proof. Take arbitrary $n=0,1, \ldots, L$ with $L>0$. Using Taylor's theorem, by straightforward calculation, we obtain for all $x \in[0,1]:$

$\frac{\bar{w}\left(t_{n+1}, x\right)-\bar{w}\left(t_{n}, x\right)}{k}=\bar{w}_{t}\left(t_{n+1 / 2}, x\right)+\delta_{n} \bar{w}_{t t}\left(t_{n+1 / 2}, x\right)+\frac{1}{k} \int_{t_{n+1 / 2}}^{t_{n+1}} \frac{\left(t_{n+1}-t\right)^{2}}{2} \bar{w}_{t t t}(x, t) \mathrm{d} t+\frac{1}{k} \int_{t_{n}}^{t_{n+1 / 2}} \frac{\left(t_{n}-t\right)^{2}}{2} \bar{w}_{t t t}(x, t) \mathrm{d} t$,

with $\delta_{n}=\frac{t_{n+1}+t_{n}}{2}$.

Similary, we have

$\frac{\bar{w}_{t}\left(t_{n+1}, x\right)+\bar{w}_{t}\left(t_{n}, x\right)}{2}=\bar{w}_{t}\left(t_{n+1 / 2}, x\right)+\delta_{n} \bar{w}_{t t}\left(t_{n+1 / 2}, x\right)+\frac{1}{2} \int_{t_{n+1 / 2}}^{t_{n+1}}\left(t_{n+1}-t\right) \bar{w}_{t t t}(x, t) \mathrm{d} t-\frac{1}{2} \int_{t_{n}}^{t_{n+1 / 2}}\left(t_{n}-t\right) \bar{w}_{t t t}(x, t) \mathrm{d} t$.

Therefore, we get

$$
\frac{\bar{w}\left(t_{n+1}, x\right)-\bar{w}\left(t_{n}, x\right)}{k}=\frac{\bar{w}_{t}\left(t_{n+1}, x\right)+\bar{w}_{t}\left(t_{n}, x\right)}{2}+k Q_{1}^{n}(x)
$$

where

$$
\begin{aligned}
Q_{1}^{n}(x) & =\frac{1}{2} \int_{t_{n+1 / 2}}^{t_{n+1}} \frac{\bar{w}_{t t t}(x, t)}{k^{2}}\left(t_{n+1}-t\right)^{2} \mathrm{~d} t+\frac{1}{2} \int_{t_{n}}^{t_{n+1 / 2}} \frac{\bar{w}_{t t t}(x, t)}{k^{2}}\left(t_{n}-t\right)^{2} \mathrm{~d} t \\
& -\frac{1}{2} \int_{t_{n+1 / 2}}^{t_{n+1}} \frac{\bar{w}_{t t t}(x, t)}{k}\left(t_{n+1}-t\right) \mathrm{d} t+\frac{1}{2} \int_{t_{n}}^{t_{n+1 / 2}} \frac{\bar{w}_{t t t}(x, t)}{k}\left(t_{n}-t\right) \mathrm{d} t .
\end{aligned}
$$

We have from (84):

$$
\frac{e_{1}^{n+1}-e_{1}^{n}}{k}=\frac{w^{n+1}-\bar{w}\left(t_{n+1}, x\right)-w^{n}+\bar{w}\left(t_{n}, x\right)}{k}=\frac{v^{n+1}+v^{n}}{2}-\frac{\bar{w}\left(t_{n+1}, x\right)-\bar{w}\left(t_{n}, x\right)}{k} .
$$

Using (97), one obtains:

$$
\frac{e_{1}^{n+1}-e_{1}^{n}}{k}=\frac{e_{2}^{n+1}+e_{2}^{n}}{2}-k Q_{1}^{n}(x)
$$

Multiplying (98) by $\left(e_{2}^{n+1}-e_{2}^{n}\right)$ and integrating over [0,1], we get:

$$
\int_{0}^{1} \frac{e_{1}^{n+1}-e_{1}^{n}}{k}\left(e_{2}^{n+1}-e_{2}^{n}\right) \mathrm{d} x=\frac{1}{2} \int_{0}^{1}\left(e_{2}^{n+1}\right)^{2} \mathrm{~d} x-\frac{1}{2} \int_{0}^{1}\left(e_{1}^{n+1}\right)^{2} \mathrm{~d} x-k \int_{0}^{1}\left(e_{2}^{n+1}-e_{2}^{n}\right) Q_{1}^{n}(x) \mathrm{d} x .
$$

Furthermore, in order to rewrite the weak formulation (15), we have from Taylor's Theorem the following expressions :

$$
\begin{aligned}
& \frac{w_{t}\left(t_{n+1}, x\right)-w_{t}\left(t_{n}, x\right)}{k}=w_{t t}\left(t_{n+1 / 2}, x\right)+\delta_{n} w_{t t t}\left(t_{n+1 / 2}, x\right)+\frac{1}{2} \int_{t_{n+1 / 2}}^{t_{n+1}} \frac{\left(t_{n+1}-t\right)^{2}}{k} w_{t t t}(x, t) \mathrm{d} t+\frac{1}{2} \int_{t_{n}}^{t_{n+1 / 2}} \frac{\left(t_{n}-t\right)^{2}}{k} w_{t t t t}(x, t) \mathrm{d} t, \\
& \frac{w_{x x}\left(t_{n+1}, x\right)+w_{x x}\left(t_{n}, x\right)}{2}=w_{x x}\left(t_{n+1 / 2}, x\right)+\delta_{n} w_{t x x}\left(t_{n+1 / 2}, x\right)+\frac{1}{2} \int_{t_{n+1 / 2}}^{t_{n+1}}\left(t_{n+1}-t\right) w_{t t x x}(x, t) \mathrm{d} t-\frac{1}{2} \int_{t_{n}}^{t_{n+1 / 2}}\left(t_{n}-t\right) w_{t t x x}(x, t) \mathrm{d} t, \\
& \frac{w_{t x}\left(t_{n+1}, x\right)+w_{t x}\left(t_{n}, x\right)}{2}=w_{t x}\left(t_{n+1 / 2}, x\right)+\delta_{n} w_{t t x}\left(t_{n+1 / 2}, x\right)+\frac{1}{2} \int_{t_{n+1 / 2}}^{t_{n+1}}\left(t_{n+1}-t\right) w_{t t t x}(x, t) \mathrm{d} t-\frac{1}{2} \int_{t_{n}}^{t_{n+1 / 2}}\left(t_{n}-t\right) w_{t t t x}(x, t) \mathrm{d} t,
\end{aligned}
$$

and

$\frac{w_{x}\left(t_{n+1}, x\right)+w_{x}\left(t_{n}, x\right)}{2}=w_{x}\left(t_{n+1 / 2}, x\right)+\delta_{n} w_{t x}\left(t_{n+1 / 2}, x\right)+\frac{1}{2} \int_{t_{n+1 / 2}}^{t_{n+1}}\left(t_{n+1}-t\right) w_{t t x}(x, t) \mathrm{d} t-\frac{1}{2} \int_{t_{n}}^{t_{n+1 / 2}}\left(t_{n}-t\right) w_{t t x}(x, t) \mathrm{d} t$. 
Taking $t_{n+1 / 2}=t$ and applying the previous expressions to the weak formulation (15), we obtain after simplifications:

$$
\begin{array}{r}
\int_{0}^{1}\left(\frac{w_{t}\left(t_{n+1}, x\right)-w_{t}\left(t_{n}, x\right)}{k}\right) \phi \mathrm{d} x+\int_{0}^{1}\left(\frac{w_{x x}\left(t_{n+1}, x\right)+w_{x x}\left(t_{n}, x\right)}{2}\right) \phi_{x x} \mathrm{~d} x \\
+\alpha\left(\frac{w_{t x}\left(t_{n+1}, 1\right)+w_{t x}\left(t_{n}, 1\right)}{2}\right) \phi_{x}(1)+\beta\left(\frac{w_{x}\left(t_{n+1}, 1\right)+w_{x}\left(t_{n}, 1\right)}{2}\right) \phi_{x}(1)=k Q_{2}^{n}(\phi) .
\end{array}
$$

where the functional $Q_{2}^{n}: V \rightarrow \mathbb{R}$ is defined as follows:

$Q_{2}^{n}(\phi)=$

$$
\begin{aligned}
& \int_{0}^{1}\left(\frac{1}{2} \int_{t_{n+1 / 2}}^{t_{n+1}} \frac{w_{t t t t}(t, x)}{k^{2}}\left(t_{n+1}-t\right)^{2} \mathrm{~d} t+\frac{1}{2} \int_{t_{n}}^{t_{n+1 / 2}} \frac{w_{t t t t}(t, x)}{k^{2}}\left(t_{n}-t\right)^{2} \mathrm{~d} t\right) \phi \mathrm{d} x \\
+ & \int_{0}^{1}\left(\frac{1}{2} \int_{t_{n+1 / 2}}^{t_{n+1}} \frac{w_{t t x x}(t, x)}{k}\left(t_{n+1}-t\right) \mathrm{d} t-\frac{1}{2} \int_{t_{n}}^{t_{n+1 / 2}} \frac{w_{t t x x}(t, x)}{k}\left(t_{n}-t\right) \mathrm{d} t\right) \phi_{x x} \mathrm{~d} x \\
+ & \beta\left(\frac{1}{2} \int_{t_{n+1 / 2}}^{t_{n+1}} \frac{w_{t t x}(t, 1)}{k}\left(t_{n+1}-t\right) \mathrm{d} t-\frac{1}{2} \int_{t_{n}}^{t_{n+1 / 2}} \frac{w_{t t x}(t, 1)}{k}\left(t_{n}-t\right) \mathrm{d} t\right) \phi_{x}(1) \\
+ & \alpha\left(\frac{1}{2} \int_{t_{n+1 / 2}}^{t_{n+1}} \frac{w_{t t t x}(t, 1)}{k}\left(t_{n+1}-t\right) \mathrm{d} t-\frac{1}{2} \int_{t_{n}}^{t_{n+1 / 2}} \frac{w_{t t t x}(t, 1)}{k}\left(t_{n}-t\right) \mathrm{d} t\right) \phi_{x}(1) .
\end{aligned}
$$

Furthermore, using (100) and (85), one obtains:

$$
\begin{aligned}
& \int_{0}^{1} \frac{e_{2}^{n+1}-e_{2}^{n}}{k} \phi_{h} \mathrm{~d} x+\int_{0}^{1} \frac{e_{1 x x}^{n+1}+e_{1 x x}^{n}}{2}\left(\phi_{h}\right)_{x x}+\alpha \frac{e_{2 x}^{n+1}(1)+e_{2 x}^{n}(1)}{2}\left(\phi_{h}\right)_{x}(1) \\
&+\beta \frac{e_{1 x}^{n+1}(1)+e_{1 x}^{n}(1)}{2}\left(\phi_{h}\right)_{x}(1) \mathrm{d} x=-k Q_{2}^{n}\left(\phi_{h}\right)+Q_{3}^{n}\left(\phi_{h}\right)
\end{aligned}
$$

where

$$
Q_{3}^{n}\left(\phi_{h}\right)=\int_{0}^{1} \frac{w_{t}^{e}\left(t_{n+1}, x\right)-\bar{w}_{t}^{e}\left(t_{n}, x\right)}{k} \phi_{h} \mathrm{~d} x+\alpha \frac{w_{t x}^{e}\left(t_{n+1}, 1\right)-\bar{w}_{t x}^{e}\left(t_{n}, 1\right)}{2}\left(\phi_{h}\right)_{x}(1) .
$$

Using (98) and taking also $\phi_{h}=k \frac{e_{2}^{n+1}+e_{2}^{n}}{2} \in V^{h}$ in (102), we obtain:

$$
\left\|y_{e}^{n+1}\right\|^{2}-\left\|y_{e}^{n}\right\|^{2}=-k^{2} \int_{0}^{1} \frac{e_{1 x x}^{n+1}+e_{1 x x}^{n}}{2}\left(Q_{1}^{n}\right)_{x x} \mathrm{~d} x-\beta k^{2} \frac{e_{1 x}^{n+1}(1)+e_{1 x}^{n}(1)}{2}\left(Q_{1}^{n}\right)_{x}(1)-\frac{k^{2}}{2} Q_{2}^{n}\left(e_{2}^{n+1}+e_{2}^{n}\right)+\frac{k}{2} Q_{3}^{n}\left(e_{2}^{n+1}+e_{2}^{n}\right) .
$$

We have the following estimate:

$$
\left\|Q_{1}^{n}\right\|_{H^{2}} \leq M k \int_{t_{n}}^{t_{n+1}}\left\|w_{t t t}(t)\right\|_{H^{2}}^{2} \mathrm{~d} t .
$$

In order to obtain the estimate of $Q_{2}^{n}$, we need to rewrite the second term of $Q_{2}^{n}\left(e_{2}^{n}\right)$. Integrating twice by parts over $[0,1]$ and assuming that $e_{2}^{n}(0)=e_{2 x}^{n}(0)=0$, we have:

$$
\begin{aligned}
& \int_{0}^{1}\left(\frac{1}{2} \int_{t_{n+1 / 2}}^{t_{n+1}} \frac{w_{t t x x}(t, x)}{k}\left(t_{n+1}-t\right) \mathrm{d} t-\frac{1}{2} \int_{t_{n}}^{t_{n+1 / 2}} \frac{w_{t t x x}(t, x)}{k}\left(t_{n}-t\right) \mathrm{d} t\right) e_{2 x x}^{n} \mathrm{~d} x \\
& =\int_{t_{n+1 / 2}}^{t_{n+1}} \frac{1}{2 k}\left(t_{n+1}-t\right)\left(w_{t t x x}(t, 1) e_{2 x}^{n}(1)-w_{t t x x x}(t, 1) e_{2}^{n}(1)+\int_{0}^{1} w_{t t x x x x}(t, x) e_{2}^{n} \mathrm{~d} x\right) \mathrm{d} t \\
& -\int_{t_{n}}^{t_{n+1 / 2}} \frac{1}{2 k}\left(t_{n}-t\right)\left(w_{t t x x}(t, 1) e_{2 x}^{n}(1)-w_{t t x x x}(t, 1) e_{2}^{n}(1)+\int_{0}^{1} w_{t t x x x x}(t, x) e_{2}^{n} \mathrm{~d} x\right) \mathrm{d} t
\end{aligned}
$$

Then,

$$
\left|Q_{2}^{n}\left(e_{2}^{n+1}+e_{2}^{n}\right)\right| \leq M\left(k \int_{t_{n}}^{t_{n+1}}\left\|w_{t t t t}(t)\right\|_{H^{2}}^{2}+\left\|w_{t t t}(t)\right\|_{H^{2}}^{2}+\left\|w_{t t}(t)\right\|_{H^{4}}^{2} \mathrm{~d} t+\left\|e_{2}^{n+1}+e_{2}^{n}\right\|_{L^{2}}^{2}+\left|e_{2 x}^{n+1}(1)+e_{2 x}^{n}(1)\right|^{2}\right) .
$$

Moreover, we have

$$
\left|Q_{3}^{n}\left(e_{2}^{n+1}+e_{2}^{n}\right)\right| \leq M\left(\frac{1}{k} \int_{t_{n}}^{t_{n+1}}\left\|w_{t t}^{e}(t)\right\|_{L^{2}}^{2} \mathrm{~d} t+\left\|w_{t}^{e}\right\|_{\left.C\left(t_{n}, t_{n+1}\right], H^{2}\right)}^{2}+\left\|e_{2}^{n+1}+e_{2}^{n}\right\|_{L^{2}}^{2}+\left|e_{2 x}^{n+1}(1)+e_{2 x}^{n}(1)\right|^{2}\right) .
$$


We deduce that

$\left\|y_{e}^{n+1}\right\|^{2}-\left\|y_{e}^{n}\right\|^{2} \leq M\left(k\left(\left\|y_{e}^{n+1}\right\|^{2}+\left\|y_{e}^{n}\right\|^{2}+\left\|w_{t}^{e}\right\|_{C\left(\left[t_{n}, t_{n+1}\right], H^{2}\right)}^{2}\right)+\int_{t_{n}}^{t_{n+1}}\left\|w_{t t}^{e}(t)\right\|_{L^{2}}^{2} \mathrm{~d} t+k^{4} \int_{t_{n}}^{t_{n+1}}\left\|w_{t t}(t)\right\|_{H^{4}}^{2}+\left\|w_{t t t}(t)\right\|_{H^{2}}^{2}+\left\|w_{t t t t}(t)\right\|_{H^{2}}^{2} \mathrm{~d} t\right)$.

Let now $m=1, \ldots, L$. Summing on $n=0, \ldots, m$, taking $k \leq \frac{1}{2 M}$, we obtain finally using Gronwall's inequality and the estimates (93)-(95), we get:

$\left\|y_{e}^{m+1}\right\|^{2} \leq M\left[\left\|y_{e}^{0}\right\|^{2}+h^{4}\left(\left\|w_{t}\right\|_{C\left([0, T] ; H^{4}\right)}^{2}+\left\|w_{t t}\right\|_{L^{2}\left([0, T] ; H^{4}\right)}^{2}\right)+k^{4}\left(\left\|w_{t t}(t)\right\|_{L^{2}\left([0, T] ; H^{4}\right)}^{2}+\left\|w_{t t t}(t)\right\|_{L^{2}\left([0, T] ; H^{2}\right)}^{2}+\left\|w_{t t t t}(t)\right\|_{L^{2}\left([0, T] ; H^{2}\right)}^{2}\right)\right]$.

Using the triangle inequality, we obtain (96).

Remark 6 The order of convergence both in time and space for the fully discrete scheme is 2 .

\section{Acknowledgements}

We are grateful to the anonymous referees whose suggestions helped us to improve the quality of the paper.

\section{References}

Adams, R. A. (1975). Sobolev spaces. Pure and Applied Mathematics, Academic Press, 65, New York-London.

Banks, H. T., \& Rosen, I. G. (1985). A Galerkin method for the estimation of parameters in hybrid systems governing the vibration of flexible beams with tip bodies. National Aeronautics and Space Administration Langley Research Center, Institute for Computer Applications in Science and Engineering. NASA Document ID: 19850011424; NASA Report/Patent No: NASA-CR-172537, ICASE Report No: 85-7.

Banks, H. T., \& Rosen, I. G. (1987). Computational methods for the identification of spatially varying stiffness and damping in beams. Control, theory and advanced technology, 3(1), 1-32.

Brenner, S. C., \& Scott, L. R. (2008). The Mathematical Theory of Finite Element Methods. Third ed. Springer, New York. https://doi.org/10.1007/978-0-387-75934-0

Brezis, H. (2011). Fonctional Analysis, Sobolev Spaces and Partial Differential Equations. Springer, New York.

Choo, S. M., Chung, S. K., \& Kannan, R. (2002). Finite element Galerkin solutions for the strongly damped extensible beam equations. Korean Journal of Computational and Applied Mathematics. 9(1), 27-43.

Evans, L. C. (1998). Partial Differential Equations. American Mathematical Society. Providence.

Guo, B. Z. (2002). Riesz basis property and exponential stability of controlled Euler-Bernoulli beam equations with variable coefficients. SIAM Journal on Control and Optimization, 40, 1905-1923. https://doi.org/10.1137/S0363012900372519

Johnson, C. (1987). Numerical solution of partial differential equations by the finite element method. Cambridge University Press.

Li, X. (2009). Observation et Commande de Quelques Systèmes à Paramètres Distribués, Automatique/Robotique, Université Claude Bernard-Lyon I.

Lions, J. L., \& Magenes, E. (1968). Problèmes aux limites non homogènes et Applications. 1, Dunod.

Miletic, M. (2015). Stability analysis and a dissipative FEM for an Euler-Bernoulli beam with tip body and passivitybased boundary control, Ph.D. Thesis, Vienna University of Technology.

Quarteroni, A., Saleri, F., \& Gervasio, P. (2010). Calcul scientifique, Cours, exercices corrigés et illustrations en MATLAB et Octave. Deuxième édition, Springer.

Shkalikov, A. (1986). Boundary problem for ordinary differential operators with parameter in boundary conditions. Journal of Soviet Mathematics. 33, 1311-1342. https://doi.org/10.1007/BF01084754

Strang, G., Fix, G.(1973). An analysis of the finite element method. Prentice-Hall, Englewood Cliffs, N. J.

Temam, R. (1988). Infinite-dimensional Dynamical Systems In Mechanics And Physics. Applied Mathematical Sciences, 68. Springer-Verlag, New York. https://doi.org/10.1007/978-1-4684-0313-8

Touré, K. A., Koua, B. J. C., \& Diop, F. N. (2016) Riesz basis property and exponential stability of a flexible EulerBernoulli beams with a force control in rotation and velocity Rotation. Far East J. Appl. Math., 99(1), 33-60. 


\section{Copyrights}

Copyright for this article is retained by the author(s), with first publication rights granted to the journal.

This is an open-access article distributed under the terms and conditions of the Creative Commons Attribution license (http://creativecommons.org/licenses/by/4.0/). 